\title{
Alkynyl Acylammoniums as Electrophilic 3C Synthons in a Formal [3+3] Annulation: Access to Functionalized 4H-Pyran-4-ones
}

Shuding Dong, Chao Fang, Weifang Tang, Tao Lu* and Ding Du*

State Key Laboratory of Natural Medicines, Department of Organic Chemistry, China Pharmaceutical University, Nanjing, 210009, P. R. China.

E-mail: lut163@163.com and ddmn9999@cpu.edu.cn

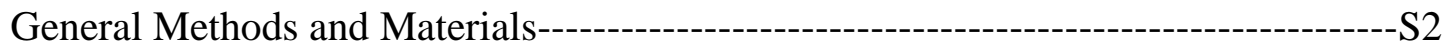

General procedure for the synthesis of $\gamma$-pyrones $\mathbf{3 / 5}$ or $\alpha$-pyrones 4 -----------------S2

Procedure for the synthesis of compound 6 from $3 \mathbf{a}$ or $\mathbf{3 g}----------------------------S 9$

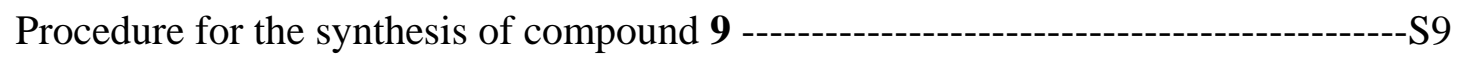

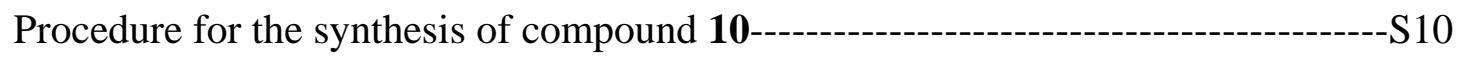

Procedure for the synthesis of compound 11----------------------------------------------S11

Procedure for the synthesis of product $\mathbf{3 g}$ from acid chloride--------------------------S11

Procedure for the reaction of alkynyl acyl imidazole 12 with $\mathbf{2 g - - - - - - - - - - - - - - - - - - - - - - S 1 2}$

Copies of NMR spectra for products 3------------------------------------------------------S13

Copy of NMR spectra for products 4 ------------------------------------------------------S36

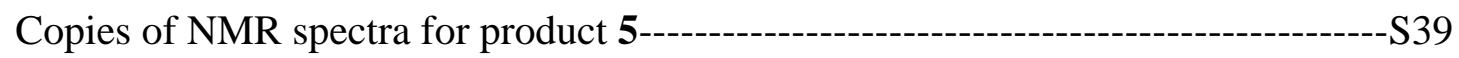

Copies of NMR spectra for compound 6------------------------------------------------S40

Copies of NMR spectra for compound 9--------------------------------------------------S41

Copies of NMR spectra for compound 10-------------------------------------------------S42

Copies of NMR spectra for compound 11--------------------------------------------------S43 
General Methods and Materials. All reactions were carried out in dry glassware, and were monitored by analytical thin-layer chromatography (TLC), which was visualized by ultraviolet light $(254 \mathrm{~nm})$. All solvents were obtained from commercial sources and were purified according to standard procedures. Alkynyl carboxylic acids $\mathbf{1}^{[1]}$ and substrates $\mathbf{2}^{[2]}$ are commercially available or prepared according to known procedures. Compound $\mathbf{1 2}$ was prepared from acyl chloride and imidazole according to the known procedure. ${ }^{[3]}$ Purification of the products was accomplished by flash chromatography using silica gel (200-300 mesh). All NMR spectra were recorded on Bruker spectrometers, running at $300 \mathrm{MHz}$ or $500 \mathrm{MHz}$ for ${ }^{1} \mathrm{H}$ and $75 \mathrm{MHz}$ or 125 $\mathrm{MHz}$ for ${ }^{13} \mathrm{C}$ respectively. Chemical shifts $(\delta)$ and coupling constants $(J)$ are reported in $\mathrm{ppm}$ and $\mathrm{Hz}$ respectively. The solvent signals were used as references (residual $\mathrm{CHCl}_{3}$ in $\left.\mathrm{CDCl}_{3}: \delta_{\mathrm{H}}=7.26 \mathrm{ppm}, \delta_{\mathrm{c}}=77.0 \mathrm{ppm}\right)$. The following abbreviations are used to indicate the multiplicity in NMR spectra: s (singlet); d (doublet); $\mathrm{t}$ (triplet); $\mathrm{q}$ (quartet); m (multiplet). High resolution mass spectrometry (HRMS) was recorded on TOF perimer for $\mathrm{ESI}^{+}$.

\section{General procedure for the synthesis of $\gamma$-pyrones $3 / 5$ or $\alpha$-pyrones 4:}

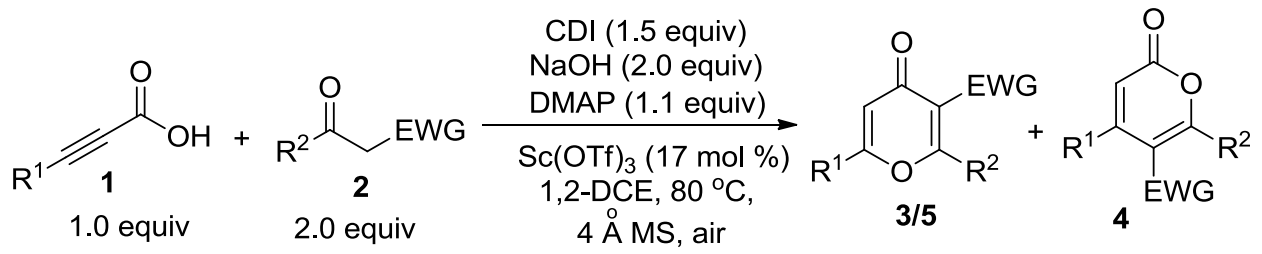

To an oven-dried $25 \mathrm{~mL}$ round-bottom flask was charged with acid 1 (0.35 mmol), substrate 2 (0.7 mmol), CDI (97 mg, $0.6 \mathrm{mmol})$, DMAP (51 mg, $0.385 \mathrm{mmol}$ ), $\mathrm{Sc}(\mathrm{OTf})_{3}(30 \mathrm{mg}, 0.06 \mathrm{mmol}), 200 \mathrm{mg}$ of $4 \AA \mathrm{MS}$ and $\mathrm{NaOH}(28 \mathrm{mg}, 0.7 \mathrm{mmol})$. Then anhydrous 1,2-DCE ( $4 \mathrm{~mL})$ was added to the flask and the resulting mixture was heated at $80{ }^{\circ} \mathrm{C}$ in air for a period time until the completion of the reaction as monitored by TLC. The mixture was cooled to room temperature. The mixture was filtered and the filtrate was concentrated under reduced pressure. The residue was purified by chromatography on silica gel using hexane/EtOAc (10:1) as the eluent to afford products $3 / 5$ or 4 .<smiles>CCOC(=O)c1c(C)oc(-c2ccccc2)cc1=O</smiles>

$3 \mathbf{a}$

Ethyl 2-methyl-4-oxo-6-phenyl-4H-pyran-3-carboxylate (3a).

$59 \mathrm{mg}, 65 \%$ yield, white solid, mp: $105-106{ }^{\circ} \mathrm{C} .{ }^{1} \mathrm{H}$ NMR (300 $\left.\mathrm{MHz} \mathrm{CDCl}_{3}\right): \delta 7.74(\mathrm{~d}, J=6.3 \mathrm{~Hz}, 2 \mathrm{H}), 7.44-7.54(\mathrm{~m}, 3 \mathrm{H}), 6.77$ 
$(\mathrm{s}, 1 \mathrm{H}), 4.40(\mathrm{q}, J=6.9 \mathrm{~Hz}, 2 \mathrm{H}), 2.49(\mathrm{~s}, 3 \mathrm{H}), 1.38(\mathrm{t}, J=6.9 \mathrm{~Hz}, 3 \mathrm{H}) .{ }^{13} \mathrm{C} \mathrm{NMR}(75$ $\left.\mathrm{MHz}, \mathrm{CDCl}_{3}\right): \delta 175.7,165.4,164.5,162.8,131.4,130.5,128.9,125.6,121.6,111.0$, 61.6, 18.5, 14.0. HRMS (ESI) calcd for $\mathrm{C}_{15} \mathrm{H}_{15} \mathrm{O}_{4}(\mathrm{M}+\mathrm{H})^{+}$: 259.0965, found 259.0964 .

Methyl 2-methyl-4-oxo-6-phenyl-4H-pyran-3-carboxylate (3b). 48 mg, $56 \%$ yield,<smiles>COC(=O)c1c(C)oc(-c2ccccc2)cc1=O</smiles>
white solid, mp: $130-131{ }^{\circ} \mathrm{C} .{ }^{1} \mathrm{H}$ NMR (500 MHz, $\left.\mathrm{CDCl}_{3}\right): \delta$ $7.75(\mathrm{~d}, J=6.7 \mathrm{~Hz}, 2 \mathrm{H}), 7.47-7.54(\mathrm{~m}, 3 \mathrm{H}), 6.81(\mathrm{~s}, 1 \mathrm{H}), 3.93(\mathrm{~s}$, $3 \mathrm{H}), 2.51(\mathrm{~s}, 3 \mathrm{H}) .{ }^{13} \mathrm{C} \mathrm{NMR}\left(75 \mathrm{MHz}, \mathrm{CDCl}_{3}\right): \delta 175.8,166.2$, $165.1,162.9,131.6,130.6,129.1,125.7,121.3,111.2,52.6,18.8$. HRMS (ESI) calcd for $\mathrm{C}_{14} \mathrm{H}_{13} \mathrm{O}_{4}(\mathrm{M}+\mathrm{H})^{+}:$245.0808, found 245.0805.

tert-Butyl 2-methyl-4-oxo-6-phenyl-4H-pyran-3-carboxylate (3c). $41 \mathrm{mg}, 41 \%$<smiles>CCCCOC(=O)c1c(C)oc(-c2ccccc2)cc1=O</smiles>
yield, white solid, mp: $145-146{ }^{\circ} \mathrm{C} .{ }^{1} \mathrm{H}$ NMR (300 MHz, $\left.\mathrm{CDCl}_{3}\right)$ : $\delta$ 7.71-7.74 (m, 2H), 7.42-7.51 (m, 3H), $6.72(\mathrm{~s}, 1 \mathrm{H}), 2.45(\mathrm{~s}$, $3 \mathrm{H}), 1.59$ (s, 9H). ${ }^{13} \mathrm{C} \mathrm{NMR}\left(75 \mathrm{MHz}, \mathrm{CDCl}_{3}\right): \delta 175.9,164.1$, $163.8,162.8,131.4,130.8,129.0,123.0,111.3,82.8,28.1,18.4$. HRMS (ESI) calcd for $\mathrm{C}_{17} \mathrm{H}_{19} \mathrm{O}_{4}(\mathrm{M}+\mathrm{H})^{+}:$287.1278, found 287.1281.

Benzyl 2-methyl-4-oxo-6-phenyl-4H-pyran-3-carboxylate (3d). 65 mg, 58\% yield,<smiles>Cc1oc(-c2ccccc2)cc(=O)c1C(=O)OCc1ccccc1</smiles>
white solid, mp: $123-124{ }^{\circ} \mathrm{C} .{ }^{1} \mathrm{H}$ NMR (300 $\left.\mathrm{MHz}, \mathrm{CDCl}_{3}\right): \delta$ 7.73-7.75 (m, 2H), 7.46-7.54 (m, 5H), 7.37-7.40 (m, 2H), 7.33 $(\mathrm{m}, 1 \mathrm{H}), 6.81(\mathrm{~s}, 1 \mathrm{H}), 5.38(\mathrm{~s}, 2 \mathrm{H}), 2.44(\mathrm{~s}, 3 \mathrm{H}) .{ }^{13} \mathrm{C}$ NMR $(75$ $\left.\mathrm{MHz}, \mathrm{CDCl}_{3}\right): \delta 175.8,165.9,164.5,162.9,135.4,131.6,130.6$, 129.1, 128.6, 128.3, 125.8, 121.4, 111.2, 67.4, 18.7. HRMS (ESI) calcd for $\mathrm{C}_{20} \mathrm{H}_{17} \mathrm{O}_{4}$ $(\mathrm{M}+\mathrm{H})^{+}: 321.1121$, found 321.1118 .

Ethyl 2-ethyl-4-oxo-6-phenyl-4H-pyran-3-carboxylate (3e). 48 mg, 50\% yield,<smiles>CCOC(=O)c1c(CC)oc(-c2ccccc2)cc1=O</smiles>

3e colorless liquid. ${ }^{1} \mathrm{H}$ NMR $\left(500 \mathrm{MHz}, \mathrm{CDCl}_{3}\right): \delta 7.79-7.78(\mathrm{~m}$, $2 \mathrm{H}), 7.58-7.52(\mathrm{~m}, 3 \mathrm{H}), 6.81(\mathrm{~s}, 1 \mathrm{H}), 4.43(\mathrm{q}, J=7.1 \mathrm{~Hz}, 2 \mathrm{H})$, $2.81(\mathrm{q}, J=7.6 \mathrm{~Hz}, 2 \mathrm{H}), 1.41(\mathrm{t}, J=7.3 \mathrm{~Hz}, 6 \mathrm{H}) .{ }^{13} \mathrm{C} \mathrm{NMR}(125$ $\left.\mathrm{MHz}, \mathrm{CDCl}_{3}\right): \delta 175.79,168.79,164.26,162.71,131.31,130.46$, 128.82, 125.41, 120.81, 110.68, 61.41, 25.73, 13.84, 11.38. HRMS (ESI) calcd for $\mathrm{C}_{16} \mathrm{H}_{17} \mathrm{O}_{4}(\mathrm{M}+\mathrm{H})^{+}:$273.1121, found 273.1125. 
Ethyl 4-oxo-2,6-diphenyl-4H-pyran-3-carboxylate (3f). $78 \mathrm{mg}, 70 \%$ yield, $\overbrace{\mathbf{3 f}}^{C_{P h}} C_{2}$ colorless liquid. ${ }^{1} \mathrm{H} \mathrm{NMR}\left(500 \mathrm{MHz}, \mathrm{CDCl}_{3}\right): \delta 7.81(\mathrm{~d}, J=6.5$ $\mathrm{Hz}, 2 \mathrm{H}), 7.73(\mathrm{~d}, J=7.5 \mathrm{~Hz}, 2 \mathrm{H}), 7.50-7.58(\mathrm{~m}, 6 \mathrm{H}), 6.93(\mathrm{~s}, 1 \mathrm{H})$, $4.28(\mathrm{q}, J=7.0 \mathrm{~Hz}, 2 \mathrm{H}), 1.17(\mathrm{t}, J=7.0 \mathrm{~Hz}, 3 \mathrm{H}) .{ }^{13} \mathrm{C} \mathrm{NMR}(75$ $\left.\mathrm{MHz}_{\mathrm{CDCl}}\right): \delta 176.0,164.3,163.0,161.9,131.4,131.2,131.0$, 130.3, 128.8, 128.5, 127.5, 125.5, 121.4, 110.5, 61.5, 13.4. HRMS (ESI) calcd for $\mathrm{C}_{20} \mathrm{H}_{17} \mathrm{O}_{4}(\mathrm{M}+\mathrm{H})^{+}:$321.1121, found 321.1117.

Methyl 4-oxo-2,6-diphenyl-4H-pyran-3-carboxylate (3g). $84 \mathrm{mg}, 78 \%$ yield, white<smiles>CC(=O)c1c(-c2ccccc2)oc(-c2ccccc2)cc1=O</smiles>

$3 g$ solid, mp: $153-154{ }^{\circ} \mathrm{C} .{ }^{1} \mathrm{H}$ NMR $\left(300 \mathrm{MHz}, \mathrm{CDCl}_{3}\right): \delta 7.81(\mathrm{~d}, J$ $=6.6 \mathrm{~Hz}, 2 \mathrm{H}), 7.71(\mathrm{~d}, J=6.9 \mathrm{~Hz}, 2 \mathrm{H}), 7.52-7.57(\mathrm{~m}, 6 \mathrm{H}), 6.90$ $(\mathrm{s}, 1 \mathrm{H}), 3.80(\mathrm{~s}, 3 \mathrm{H}) .{ }^{13} \mathrm{C} \mathrm{NMR}\left(75 \mathrm{MHz}, \mathrm{CDCl}_{3}\right): \delta 176.3,165.2$, $163.4,162.4,131.7,131.6,131.4,130.7,129.1,128.9,127.7$, 125.9, 121.5, 110.9, 52.7. HRMS (ESI) calcd for $\mathrm{C}_{19} \mathrm{H}_{15} \mathrm{O}_{4}(\mathrm{M}+\mathrm{H})^{+}: 307.0965$, found 307.0962 .

Methyl 2-(4-chlorophenyl)-4-oxo-6-phenyl-4H-pyran-3-carboxylate (3h). $73 \mathrm{mg}$,<smiles>CC(=O)c1c(-c2ccc(Cl)cc2)oc(-c2ccccc2)cc1=O</smiles>
$61 \%$ yield, white solid, mp: $128-129{ }^{\circ} \mathrm{C} .{ }^{1} \mathrm{H}$ NMR (300 MHz, $\left.\mathrm{CDCl}_{3}\right): \delta 7.79(\mathrm{~d}, J=6.6 \mathrm{~Hz}, 2 \mathrm{H}), 7.65(\mathrm{~d}, J=8.4 \mathrm{~Hz}, 2 \mathrm{H})$, 7.49-7.54 (m, 5H), $6.89(\mathrm{~s}, 1 \mathrm{H}), 3.82(\mathrm{~s}, 3 \mathrm{H}) .{ }^{13} \mathrm{C}$ NMR $(75$ $\left.\mathrm{MHz}, \mathrm{CDCl}_{3}\right): \delta 176.1,165.1,163.5,161.2,138.0,131.8$, 130.6, 129.8, 129.3, 129.2, 129.1, 125.9, 121.7, 111.0, 52.9. HRMS (ESI) calcd for $\mathrm{C}_{19} \mathrm{H}_{14} \mathrm{ClO}_{4}(\mathrm{M}+\mathrm{H})^{+}:$341.0575, found 341.0578.

Methyl 4-oxo-6-phenyl-2-(p-tolyl)-4H-pyran-3-carboxylate (3i). 93 mg, 83\% yield,<smiles>CC(=O)c1c(-c2ccc(C)cc2)oc(-c2ccccc2)cc1=O</smiles>
white solid, mp: $137-138{ }^{\circ} \mathrm{C} .{ }^{1} \mathrm{H}$ NMR (300 MHz, $\left.\mathrm{CDCl}_{3}\right): \delta$ $7.81(\mathrm{~d}, J=6.9 \mathrm{~Hz}, 2 \mathrm{H}), 7.61(\mathrm{~d}, J=8.1 \mathrm{~Hz}, 2 \mathrm{H}), 7.50-7.53$ $(\mathrm{m}, 3 \mathrm{H}), 7.32(\mathrm{~d}, J=7.8 \mathrm{~Hz}, 2 \mathrm{H}), 6.89(\mathrm{~s}, 1 \mathrm{H}), 3.81(\mathrm{~s}, 3 \mathrm{H})$, $2.44(\mathrm{~s}, 3 \mathrm{H}) .{ }^{13} \mathrm{C}$ NMR $\left(75 \mathrm{MHz}, \mathrm{CDCl}_{3}\right): \delta$ 176.4, 165.5, $163.3,162.6,142.2,131.7,130.8,129.7,129.1,128.5,127.7,125.9,121.0,110.8$, 52.8, 21.5. HRMS (ESI) calcd for $\mathrm{C}_{20} \mathrm{H}_{17} \mathrm{O}_{4}(\mathrm{M}+\mathrm{H})^{+}$: 321.1121 , found 321.1121 .

3-Acetyl-2-methyl-6-phenyl-4H-pyran-4-one (3j). $29 \mathrm{mg}, 36 \%$ yield, white solid, mp: $140-141{ }^{\circ} \mathrm{C} .{ }^{1} \mathrm{H}$ NMR (500 MHz, $\left.\mathrm{CDCl}_{3}\right): \delta 7.76(\mathrm{~d}, J=7.3 \mathrm{~Hz}, 2 \mathrm{H}), 7.56-7.46$ 
<smiles>CC(=O)c1c(C)oc(-c2ccccc2)cc1=O</smiles>

$(\mathrm{m}, 3 \mathrm{H}), 6.78(\mathrm{~s}, 1 \mathrm{H}), 2.61(\mathrm{~s}, 3 \mathrm{H}), 2.50(\mathrm{~s}, 3 \mathrm{H}) .{ }^{13} \mathrm{C} \mathrm{NMR}$ $\left(125 \mathrm{MHz}, \mathrm{CDCl}_{3}\right): \delta 200.25,177.49,167.22,162.71,131.60$, $130.57,129.08,126.93,125.77,111.80,31.93,18.87$. HRMS (ESI) calcd for $\mathrm{C}_{14} \mathrm{H}_{13} \mathrm{O}_{3}(\mathrm{M}+\mathrm{H})^{+}:$229.0859, found 229.0864 .

3-Benzoyl-2,6-diphenyl-4H-pyran-4-one (3k). $38 \mathrm{mg}, 31 \%$ yield, white solid, mp:<smiles>O=C(c1ccccc1)c1c(-c2ccccc2)oc(-c2ccccc2)cc1=O</smiles>
183-184 ${ }^{\circ} \mathrm{C} .{ }^{1} \mathrm{H}$ NMR $\left(500 \mathrm{MHz}, \mathrm{CDCl}_{3}\right): \delta 7.93(\mathrm{~d}, J=7.7 \mathrm{~Hz}$, $2 \mathrm{H}), 7.87(\mathrm{~d}, J=6.8 \mathrm{~Hz}, 2 \mathrm{H}), 7.63(\mathrm{~d}, J=7.5 \mathrm{~Hz}, 2 \mathrm{H}), 7.57-7.52$ (m, 4H), 7.44-7.40 (m, 3H), $7.37(\mathrm{t}, J=7.5 \mathrm{~Hz}, 2 \mathrm{H}), 6.93(\mathrm{~s}, 1 \mathrm{H})$. ${ }^{13} \mathrm{C}$ NMR $\left(125 \mathrm{MHz}, \mathrm{CDCl}_{3}\right): \delta 193.13,177.89,163.59,161.60$, $136.58,133.80,131.69,131.27,131.18,130.84$, 129.30, 129.16, 128.80, 128.69, 128.19, 126.14, 125.91, 110.98. HRMS (ESI) calcd for $\mathrm{C}_{24} \mathrm{H}_{17} \mathrm{O}_{3}(\mathrm{M}+\mathrm{H})^{+}: 353.1172$, found 353.1178 .

Methyl 6-(4-fluorophenyl)-4-oxo-2-phenyl-4H-pyran-3-carboxylate (3I). 52 mg,<smiles>CC(=O)c1c(-c2ccccc2)oc(-c2ccc(F)cc2)cc1=O</smiles>
$46 \%$ yield, white solid, mp: $166-167{ }^{\circ} \mathrm{C} .{ }^{1} \mathrm{H}$ NMR (500 $\left.\mathrm{MHz}, \mathrm{CDCl}_{3}\right): \delta$ 7.80-7.82 (m, 2H), 7.69-7.71 (m, 2H), $7.57(\mathrm{~m}, 1 \mathrm{H}), 7.50-7.53(\mathrm{~m}, 2 \mathrm{H}), 7.20(\mathrm{t}, J=8.5 \mathrm{~Hz}, 2 \mathrm{H})$, $6.84(\mathrm{~s}, 1 \mathrm{H}), 3.80(\mathrm{~s}, 3 \mathrm{H}) .{ }^{13} \mathrm{C} \mathrm{NMR}\left(125 \mathrm{MHz}, \mathrm{CDCl}_{3}\right): \delta$ $176.14,165.12,164.76(\mathrm{~d}, J=252.3 \mathrm{~Hz}), 162.42,131.62,131.26,128.95,128.16$ (d, $J=8.6 \mathrm{~Hz}), 127.72,126.93(\mathrm{~d}, J=2.1 \mathrm{~Hz}), 121.48,116.44(\mathrm{~d}, J=22.2 \mathrm{~Hz}), 110.67$, 52.74. HRMS (ESI) calcd for $\mathrm{C}_{19} \mathrm{H}_{14} \mathrm{FO}_{4}(\mathrm{M}+\mathrm{H})^{+}$: 325.0871 , found 325.0869 .

Methyl 6-(4-chlorophenyl)-4-oxo-2-phenyl-4H-pyran-3-carboxylate (3m). $73 \mathrm{mg}$,<smiles>CC(=O)c1c(-c2ccccc2)oc(-c2ccc(Cl)cc2)cc1=O</smiles>
$61 \%$ yield, white solid, mp: $170-171{ }^{\circ} \mathrm{C} .{ }^{1} \mathrm{H}$ NMR (500 $\left.\mathrm{MHz}, \mathrm{CDCl}_{3}\right): \delta 7.74(\mathrm{~d}, J=8.5 \mathrm{~Hz}, 2 \mathrm{H}), 7.69(\mathrm{~d}, J=7.5$ $\mathrm{Hz}, 2 \mathrm{H}), 7.57$ (t, $J=7.0 \mathrm{~Hz}, 1 \mathrm{H}), 7.48-7.54(\mathrm{~m}, 4 \mathrm{H}), 6.86$ (s, 1H), $3.79(\mathrm{~s}, 3 \mathrm{H}) \cdot{ }^{13} \mathrm{C}$ NMR (125 $\left.\mathrm{MHz}, \mathrm{CDCl}_{3}\right): \delta$ $176.06,165.05,162.42,162.24,138.03,131.65,131.18,129.48,129.15,128.95$, 127.70, 127.16, 121.56, 111.00, 52.74. HRMS (ESI) calcd for $\mathrm{C}_{19} \mathrm{H}_{14} \mathrm{ClO}_{4}(\mathrm{M}+\mathrm{H})^{+}$: 341.0575, found 341.0570.

Methyl 6-(4-bromophenyl)-4-oxo-2-phenyl-4H-pyran-3-carboxylate (3n). $58 \mathrm{mg}$, $43 \%$ yield, white solid, mp: $191-192{ }^{\circ} \mathrm{C} .{ }^{1} \mathrm{H}$ NMR (500 MHz, $\mathrm{CDCl}_{3}$ ): $\delta 7.70-7.68(\mathrm{~m}$, 
2H), 7.66-7.64 (m, 4H), $7.58(\mathrm{t}, J=6.8 \mathrm{~Hz}, 1 \mathrm{H}), 7.53-7.51(\mathrm{~m}, 2 \mathrm{H}), 6.88(\mathrm{~s}, 1 \mathrm{H}), 3.79$<smiles>CC(=O)c1c(-c2ccccc2)oc(-c2ccc(Br)cc2)cc1=O</smiles>
(s, 3H). ${ }^{13} \mathrm{C}$ NMR (125 MHz, $\left.\mathrm{CDCl}_{3}\right): \delta 176.10,165.06$, $162.49,162.36,132.50,131.68,131.22,129.66,128.99$ $127.75,127.34,126.48,121.64,111.08,52.79$. HRMS (ESI) calcd for $\mathrm{C}_{19} \mathrm{H}_{14} \mathrm{BrO}_{4}(\mathrm{M}+\mathrm{H})^{+}$: 385.0070, found 385.0076 .

Methyl 4-oxo-2-phenyl-6-(p-tolyl)-4H-pyran-3-carboxylate (3o). 74 mg, 66\% yield,<smiles>CC(=O)c1c(-c2ccccc2)oc(-c2ccc(C)cc2)cc1=O</smiles>
white solid, mp: $149-150{ }^{\circ} \mathrm{C} .{ }^{1} \mathrm{H}$ NMR $(500 \mathrm{MHz}$, $\left.\mathrm{CDCl}_{3}\right): \delta 6.89-7.72(\mathrm{~m}, 4 \mathrm{H}), 7.50-7.57(\mathrm{~m}, 3 \mathrm{H}), 7.30(\mathrm{~d}$ $J=7.5 \mathrm{~Hz}, 2 \mathrm{H}), 6.85(\mathrm{~s}, 1 \mathrm{H}), 3.79(\mathrm{~s}, 3 \mathrm{H}), 2.43(\mathrm{~s}, 3 \mathrm{H})$. ${ }^{13} \mathrm{C}$ NMR $\left(125 \mathrm{MHz}, \mathrm{CDCl}_{3}\right): \delta 176.35,165.31,163.57$, $162.24,142.41,131.50,131.43,129.85,128.89,127.87,127.72,125.83,121.39$, 110.20, 52.69, 21.43. HRMS (ESI) calcd for $\mathrm{C}_{20} \mathrm{H}_{17} \mathrm{O}_{4}(\mathrm{M}+\mathrm{H})^{+}$: 321.1121, found 321.1118 .

Methyl 6-(3-chlorophenyl)-4-oxo-2-phenyl-4H-pyran-3-carboxylate (3p). $68 \mathrm{mg}$,<smiles>CC(=O)c1c(-c2ccccc2)oc(-c2cccc(Cl)c2)cc1=O</smiles>
57\% yield, white solid, mp: 96-97 ${ }^{\circ} \mathrm{C} .{ }^{1} \mathrm{H}$ NMR (500 $\left.\mathrm{MHz}, \mathrm{CDCl}_{3}\right): \delta 7.78(\mathrm{~s}, 1 \mathrm{H}), 7.71-7.67(\mathrm{~m}, 3 \mathrm{H}), 7.58(\mathrm{t}$, $J=7.3 \mathrm{~Hz}, 1 \mathrm{H}), 7.55-7.50(\mathrm{~m}, 3 \mathrm{H}), 7.45(\mathrm{t}, J=7.9 \mathrm{~Hz}$, $1 \mathrm{H}), 6.88(\mathrm{~s}, 1 \mathrm{H}), 3.80(\mathrm{~s}, 3 \mathrm{H}) \cdot{ }^{13} \mathrm{C}$ NMR $(125 \mathrm{MHz}$, $\left.\mathrm{CDCl}_{3}\right): \delta 176.02,165.01,162.58,161.87,135.41,132.51,131.70,131.14,130.47$, $129.00,127.75,125.96,124.05,121.71,111.59,52.78$. HRMS (ESI) calcd for $\mathrm{C}_{19} \mathrm{H}_{14} \mathrm{ClO}_{4}(\mathrm{M}+\mathrm{H})^{+}:$341.0575, found 341.0579.

Methyl 4-oxo-2-phenyl-6-(m-tolyl)-4H-pyran-3-carboxylate (3q). 49 mg, 44\%,<smiles>CC(=O)c1c(-c2ccccc2)oc(-c2cccc(C)c2)cc1=O</smiles>
yield, white solid, mp: $132-133{ }^{\circ} \mathrm{C} .{ }^{1} \mathrm{H}$ NMR $(500 \mathrm{MHz}$, $\left.\mathrm{CDCl}_{3}\right): \delta 7.71(\mathrm{~d}, J=7.2 \mathrm{~Hz}, 2 \mathrm{H}), 7.62-7.58(\mathrm{~m}, 2 \mathrm{H})$, 7.58-7.49 (m, 3H), $7.39(\mathrm{t}, J=7.6 \mathrm{~Hz}, 1 \mathrm{H}), 7.35(\mathrm{~d}, J=$ $7.5 \mathrm{~Hz}, 1 \mathrm{H}), 6.88(\mathrm{~s}, 1 \mathrm{H}), 3.80(\mathrm{~s}, 3 \mathrm{H}), 2.43(\mathrm{~s}, 3 \mathrm{H}) .{ }^{13} \mathrm{C}$ NMR (125 MHz, $\left.\mathrm{CDCl}_{3}\right): \delta 176.31,165.26,163.61,162.37,138.98,132.52,131.51$, $131.39,130.65,129.02,128.90,127.73,126.41,123.12,121.48,110.83,52.69,21.40$. HRMS (ESI) calcd for $\mathrm{C}_{20} \mathrm{H}_{17} \mathrm{O}_{4}(\mathrm{M}+\mathrm{H})^{+}:$321.1121, found 321.1124 . 
Methyl 6-(2-chlorophenyl)-4-oxo-2-phenyl-4H-pyran-3-carboxylate (3r). $50 \mathrm{mg}$,<smiles>CC(=O)c1c(-c2ccccc2)oc(-c2ccccc2Cl)cc1=O</smiles>
$42 \%$ yield, white solid, mp: $153-154{ }^{\circ} \mathrm{C} .{ }^{1} \mathrm{H}$ NMR (500 MHz, $\left.\mathrm{CDCl}_{3}\right): \delta 7.70(\mathrm{~d}, J=7.5 \mathrm{~Hz}, 2 \mathrm{H}), 7.56-7.51(\mathrm{~m}, 3 \mathrm{H}), 7.50-$ $7.44(\mathrm{~m}, 3 \mathrm{H}), 7.40(\mathrm{t}, J=7.5 \mathrm{~Hz}, 1 \mathrm{H}), 6.74(\mathrm{~s}, 1 \mathrm{H}), 3.81(\mathrm{~s}$, $3 \mathrm{H}) .{ }^{13} \mathrm{C} \mathrm{NMR}\left(125 \mathrm{MHz}, \mathrm{CDCl}_{3}\right): \delta 175.96,165.19,163.00$, $162.58,132.74,132.05,131.60,131.09,130.81,130.58,128.82,127.76,127.22$, 121.43, 116.48, 52.76. HRMS (ESI) calcd for $\mathrm{C}_{19} \mathrm{H}_{14} \mathrm{ClO}_{4}(\mathrm{M}+\mathrm{H})^{+}: 341.0575$, found 341.0575 .

Methyl 6-(naphthalen-1-yl)-4-oxo-2-phenyl-4H-pyran-3-carboxylate (3s). 34 mg,<smiles>CC(=O)c1c(-c2ccccc2)oc(-c2cccc3ccccc23)cc1=O</smiles>
$27 \%$ yield, white solid, mp: $153-154{ }^{\circ} \mathrm{C} .{ }^{1} \mathrm{H}$ NMR (500 $\left.\mathrm{MHz}, \mathrm{CDCl}_{3}\right): \delta 8.10-8.09(\mathrm{~m}, 1 \mathrm{H}), 8.03(\mathrm{~d}, J=8.2 \mathrm{~Hz}$, 1H), 7.96-7.94 (m, 1H), 7.72-7.68 (m, 3H), 7.60-7.56 (m, $3 \mathrm{H}), 7.52(\mathrm{t}, J=7.3 \mathrm{~Hz}, 1 \mathrm{H}), 7.46(\mathrm{t}, J=7.4 \mathrm{~Hz}, 2 \mathrm{H}), 6.79$ (s, 1H), 3.85 (s, 3H). ${ }^{13} \mathrm{C}$ NMR (125 MHz, $\left.\mathrm{CDCl}_{3}\right): \delta 176.12,165.34,164.96,162.89$, $133.64,131.66,131.56,131.09,130.14,129.37,128.81,128.73,127.85,127.67$, $127.55,126.60,124.95,124.32,121.48,116.46,52.77$. HRMS (ESI) calcd for $\mathrm{C}_{23} \mathrm{H}_{17} \mathrm{O}_{4}(\mathrm{M}+\mathrm{H})^{+}:$357.1121, found 357.1120.

Methyl 6-(furan-2-yl)-4-oxo-2-phenyl-4H-pyran-3-carboxylate (3t). 19 mg, 18\%<smiles>CC(=O)c1c(-c2ccccc2)oc(-c2ccco2)cc1=O</smiles>
yield, colorless liquid. ${ }^{1} \mathrm{H}$ NMR $\left(500 \mathrm{MHz}, \mathrm{CDCl}_{3}\right): \delta$ 7.69-7.65 (m, 2H), $7.61(\mathrm{~d}, J=1.0 \mathrm{~Hz}, 1 \mathrm{H}), 7.57-7.53(\mathrm{~m}, 1 \mathrm{H})$, $7.50(\mathrm{t}, J=7.4 \mathrm{~Hz}, 2 \mathrm{H}), 7.01(\mathrm{~d}, J=3.5 \mathrm{~Hz}, 1 \mathrm{H}), 6.77(\mathrm{~s}, 1 \mathrm{H})$, $6.58(\mathrm{dd}, J=3.5,1.7 \mathrm{~Hz}, 1 \mathrm{H}), 3.78(\mathrm{~s}, 3 \mathrm{H}) .{ }^{13} \mathrm{C} \mathrm{NMR}(75$ $\left.\mathrm{MHz}, \mathrm{CDCl}_{3}\right): \delta 175.78,165.16,161.79,155.31,145.91,145.42,131.62,131.20$, $128.92,127.74,121.63,112.87,112.45,108.64,52.80$. HRMS (ESI) calcd for $\mathrm{C}_{17} \mathrm{H}_{13} \mathrm{O}_{5}(\mathrm{M}+\mathrm{H})^{+}:$297.0757, found 297.0761.

Methyl 4-cyclopropyl-2-oxo-6-phenyl-2H-pyran-5-carboxylate (3u). $41 \mathrm{mg}, 43 \%$<smiles>CC(=O)c1c(-c2ccccc2)oc(C2CC2)cc1=O</smiles>
3u yield, colorless liquid. ${ }^{1} \mathrm{H} \mathrm{NMR}\left(500 \mathrm{MHz}, \mathrm{CDCl}_{3}\right): \delta 7.53(\mathrm{~d}, J$ $=8.0 \mathrm{~Hz}, 2 \mathrm{H}), 7.49(\mathrm{~d}, J=7.1 \mathrm{~Hz}, 1 \mathrm{H}), 7.44(\mathrm{t}, J=7.5 \mathrm{~Hz}, 2 \mathrm{H})$, 6.27 (s, 1H), 3.74 (s, 3H), 1.90-1.82 (m, 1H), 1.11-1.04 (m, 4H). 
${ }^{13} \mathrm{C}$ NMR (125 MHz, $\left.\mathrm{CDCl}_{3}\right): \delta$ 175.73, 169.61, 165.32, 161.72, 131.34, 131.22, 128.77, 127.41, 121.19, 111.09, 52.59, 13.83, 8.33. HRMS (ESI) calcd for $\mathrm{C}_{16} \mathrm{H}_{15} \mathrm{O}_{4}$ $(\mathrm{M}+\mathrm{H})^{+}:$271.0965, found 271.0965 .

Methyl 4-oxo-6-pentyl-2-phenyl-4H-pyran-3-carboxylate (3v). 19 mg, $18 \%$ yield,<smiles>CCCCCc1cc(=O)c(C(C)=O)c(-c2ccccc2)o1</smiles>
colorless liquid. ${ }^{1} \mathrm{H}$ NMR $\left(500 \mathrm{MHz}, \mathrm{CDCl}_{3}\right): \delta 7.60(\mathrm{~d}$, $J=7.5 \mathrm{~Hz}, 2 \mathrm{H}), 7.52(\mathrm{t}, J=7.5 \mathrm{~Hz}, 1 \mathrm{H}), 7.47(\mathrm{t}, J=7.5$ $\mathrm{Hz}, 2 \mathrm{H}), 6.26$ (s, 1H), 3.77 (s, 3H), 2.59 (t, $J=7.5 \mathrm{~Hz}$, 2H), 1.74-1.68 (m, 2H), 1.39-1.36 (m, 4H), $0.92(\mathrm{t}, J=7.0 \mathrm{~Hz}, 3 \mathrm{H}) .{ }^{13} \mathrm{C}$ NMR $(125$ $\left.\mathrm{MHz}, \mathrm{CDCl}_{3}\right): \delta 176.34,169.26,165.41,162.57,131.42,131.38,128.80,127.63$, 121.14, 113.37, 52.72, 33.52, 30.96, 26.28, 22.22, 13.79. HRMS (ESI) calcd for $\mathrm{C}_{18} \mathrm{H}_{21} \mathrm{O}_{4}(\mathrm{M}+\mathrm{H})^{+}:$301.1434, found 301.1438.

Methyl 6-methyl-4-oxo-2-phenyl-4H-pyran-3-carboxylate (3w). $34 \mathrm{mg}$, $40 \%$ yield,<smiles>CC(=O)c1c(-c2ccccc2)oc(C)cc1=O</smiles>
colorless liquid. ${ }^{1} \mathrm{H}$ NMR $\left(300 \mathrm{MHz}, \mathrm{CDCl}_{3}\right): \delta 7.62-7.59(\mathrm{~m}$, $2 \mathrm{H}), 7.75-7.43(\mathrm{~m}, 3 \mathrm{H}), 6.26(\mathrm{~s}, 1 \mathrm{H}), 3.76(\mathrm{~s}, 3 \mathrm{H}), 2.35$ (s, 3H).

${ }^{13} \mathrm{C}$ NMR (125 MHz, $\left.\mathrm{CDCl}_{3}\right): \delta 176.21,165.62,165.35,162.71$, 131.44, 131.28, 128.78, 127.66, 121.11, 114.13, 52.69, 19.82. HRMS (ESI) calcd for $\mathrm{C}_{14} \mathrm{H}_{13} \mathrm{O}_{4}(\mathrm{M}+\mathrm{H})^{+}:$245.0808, found 245.0815.

Methyl 4-methyl-2-oxo-6-phenyl-2H-pyran-5-carboxylate (4a). 20 mg, 23\% yield,<smiles>COC(=O)c1c(C)cc(=O)oc1-c1ccccc1</smiles>
white solid, mp: 99-100 ${ }^{\circ} \mathrm{C} .{ }^{1} \mathrm{H}$ NMR (500 MHz, $\left.\mathrm{CDCl}_{3}\right): \delta 7.57(\mathrm{~d}$, $J=7.4 \mathrm{~Hz}, 2 \mathrm{H}), 7.52-7.41(\mathrm{~m}, 3 \mathrm{H}), 6.16(\mathrm{~s}, 1 \mathrm{H}), 3.66(\mathrm{~s}, 3 \mathrm{H}), 2.25(\mathrm{~s}$, $3 \mathrm{H}) .{ }^{13} \mathrm{C} \mathrm{NMR}\left(125 \mathrm{MHz}, \mathrm{CDCl}_{3}\right): \delta 166.60,161.04,160.32,153.69$, 131.88, 128.52, 127.80, 113.26, 112.44, 52.46, 20.16. HRMS (ESI) calcd for $\mathrm{C}_{14} \mathrm{H}_{13} \mathrm{O}_{4}(\mathrm{M}+\mathrm{H})^{+}:$245.0808, found 245.0811 .

Methyl 2-oxo-6-phenyl-2H-pyran-5-carboxylate (4b). $39 \mathrm{mg}$, 48\% yield, colorless<smiles>CC(=O)c1ccc(=O)oc1-c1ccccc1</smiles>
liquid. ${ }^{1} \mathrm{H}$ NMR $\left(300 \mathrm{MHz}, \mathrm{CDCl}_{3}\right): \delta 7.86(\mathrm{~d}, J=9.7 \mathrm{~Hz}, 1 \mathrm{H})$, 7.58-7.43 (m, 5H), $6.34(\mathrm{~d}, J=9.7 \mathrm{~Hz}, 1 \mathrm{H}), 3.71(\mathrm{~s}, 3 \mathrm{H}) .{ }^{13} \mathrm{C} \mathrm{NMR}$ $\left(75 \mathrm{MHz}, \mathrm{CDCl}_{3}\right): \delta 167.2,164.9,160.1,144.1,132.1,131.2,129.1$, 128.0, 113.2, 109.4, 52.2. HRMS (ESI) calcd for $\mathrm{C}_{13} \mathrm{H}_{11} \mathrm{O}_{4}(\mathrm{M}+\mathrm{H})^{+}$: 231.0652, found 231.0656.

4-Oxo-2,6-diphenyl-4H-pyran-3-carbonitrile (5). $49 \mathrm{mg}, 51 \%$ yield, white solid, 
mp: $177-178{ }^{\circ} \mathrm{C} .{ }^{1} \mathrm{H}$ NMR $\left(500 \mathrm{MHz}, \mathrm{CDCl}_{3}\right): \delta 8.09(\mathrm{~d}, J=7.5 \mathrm{~Hz}$,<smiles>N#Cc1c(-c2ccccc2)oc(-c2ccccc2)cc1=O</smiles>
2H), $7.82(\mathrm{~d}, J=7.0 \mathrm{~Hz}, 2 \mathrm{H}), 7.68(\mathrm{t}, J=7.5 \mathrm{~Hz}, 1 \mathrm{H}), 7.54-7.64(\mathrm{~m}$, $5 \mathrm{H}), 6.91(\mathrm{~s}, 1 \mathrm{H}) .{ }^{13} \mathrm{C} \mathrm{NMR}\left(75 \mathrm{MHz}, \mathrm{CDCl}_{3}\right): \delta 175.1,170.3,163.7$, $133.3,132.4,129.8,129.6,129.4,129.2,128.4,126.0,113.4,109.9$, 102.0. HRMS (ESI) calcd for $\mathrm{C}_{18} \mathrm{H}_{12} \mathrm{NO}_{2}(\mathrm{M}+\mathrm{H})^{+}: 274.0863$, found 274.0859 .

Procedure for the synthesis of compound 6 from 3a or $3 g$.

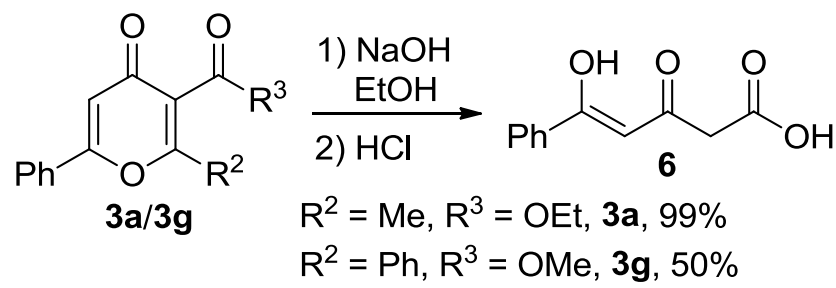

To a $10 \mathrm{~mL}$ round-bottom flask was charged with $\mathbf{3 a} / \mathbf{3 g}(0.1 \mathrm{mmol})$ and $3 \mathrm{~mL}$ of EtOH. Then $5 \mathrm{~mL}$ of $1 \mathrm{M} \mathrm{NaOH}(\mathrm{aq})$ was added and the resulting mixture was stirred for a period of time until the completion of the reaction as monitored by TLC. The solution was acidified to $\mathrm{pH}=3$ with $1 \mathrm{M} \mathrm{HCl}$, and was subsequently extracted with DCM. Then, the organic phase was dried over anhydrous $\mathrm{MgSO}_{4}$. The mixture was filtered and the filtrate was concentrated under reduced pressure. The residue was purified by chromatography on silica gel using hexane/EtOAc $(1: 2)$ as the eluent to afford product 6 .

5-Hydroxy-3-oxo-5-phenylpent-4-enoic acid (6). $20 \mathrm{mg}$ from 3a and $10 \mathrm{mg}$ from $\mathbf{3 g}$.

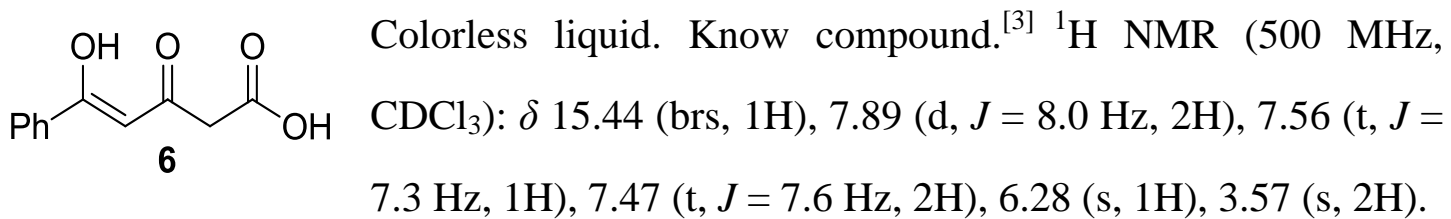

Procedure for the synthesis of compound 9.<smiles>O=C(O)c1c(-c2ccccc2)oc(-c2ccccc2)cc1=O</smiles>

To a $10 \mathrm{~mL}$ round-bottom flask was charged with $\mathbf{3 g}(31 \mathrm{mg}, 0.1 \mathrm{mmol})$ and $3 \mathrm{~mL}$ of THF. Then $5 \mathrm{~mL}$ of $1 \mathrm{M} \mathrm{NaOH}(\mathrm{aq})$ was added and the resulting mixture was stired 
for a period of time until the completion of the reaction as monitored by TLC. The solution was acidified to $\mathrm{pH}=3$ with $1 \mathrm{M} \mathrm{HCl}$, and was subsequently extracted with DCM. Then, the organic phase was dried over anhydrous $\mathrm{MgSO}_{4}$. The mixture was filtered and the filtrate was concentrated under reduced pressure. The residue was purified by chromatography on silica gel using hexane/EtOAc (1:2) as the eluent to afford $22 \mathrm{mg}$ of compound 9.

4-Oxo-2,6-diphenyl-4H-pyran-3-carboxylic acid (9). White solid, mp: 202-203 ${ }^{\circ} \mathrm{C}$.<smiles>O=C(O)c1c(-c2ccccc2)oc(-c2ccccc2)cc1=O</smiles>

${ }^{1} \mathrm{H}$ NMR $\left(300 \mathrm{MHz}, \mathrm{CDCl}_{3}\right): \delta$ 7.84-7.81 (m, 2H), 7.69-7.66 (m, $2 \mathrm{H}), 7.61-7.51(\mathrm{~m}, 6 \mathrm{H}), 7.11(\mathrm{~s}, 1 \mathrm{H}) .{ }^{13} \mathrm{C}$ NMR $(125 \mathrm{MHz}$, $\left.9 \mathrm{CDCl}_{3}\right): \delta 181.97,173.18,165.01,163.06,132.83,131.87,131.75$, 129.45, 129.31, 128.21, 126.33, 113.41, 110.17. HRMS (ESI) calcd for $\mathrm{C}_{18} \mathrm{H}_{13} \mathrm{O}_{4}$ $(\mathrm{M}+\mathrm{H})^{+}:$293.0808, found 293.0815.

Procedure for the synthesis of compound 10 from 9.

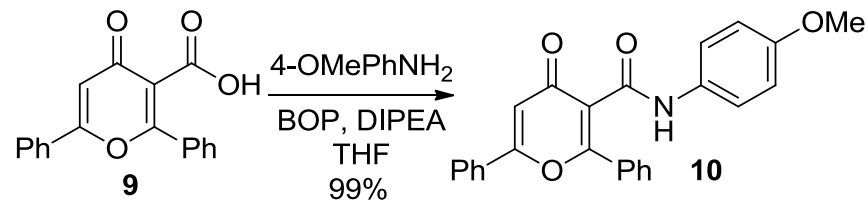

To a $10 \mathrm{~mL}$ round-bottom flask was charged with 9 (29 mg, $0.1 \mathrm{mmol})$, BOP (66 $\mathrm{mg}, 0.15 \mathrm{mmol}), 3 \mathrm{~mL}$ of THF and DIPEA (26 mg, $0.2 \mathrm{mmol})$. After $5 \mathrm{mins}$, 4- $\mathrm{OMePhNH}_{2}(15 \mathrm{mg}, 0.12 \mathrm{mmol})$ was added to the solution, and the resulting mixture was stired for a period of time until the completion of the reaction as monitored by TLC. Then, the reaction mixture was concentrated under reduced pressure. The residue was purified by chromatography on silica gel using hexane/EtOAc (3:1) as the eluent to afford $39 \mathrm{mg}$ of compound $\mathbf{1 0 .}$

$N$-(4-methoxyphenyl)-4-oxo-2,6-diphenyl-4H-pyran-3-carboxamide (10). White<smiles>COc1ccc(NC(=O)c2c(-c3ccccc3)oc(-c3ccccc3)cc2=O)cc1</smiles>
solid, mp: $177-178{ }^{\circ} \mathrm{C} .{ }^{1} \mathrm{H}$ NMR $\left(500 \mathrm{MHz}, \mathrm{CDCl}_{3}\right): \delta$ 10.94 (brs, 1H), 7.82 (d, $J=7.5 \mathrm{~Hz}, 2 \mathrm{H}), 7.68$ (d, $J=$ 7.0 Hz, 2H), 7.49-7.57 (m, 8H), $6.99(\mathrm{~s}, 1 \mathrm{H}), 6.84(\mathrm{~d}$, $J=9.0 \mathrm{~Hz}, 2 \mathrm{H}), 3.78(\mathrm{~s}, 3 \mathrm{H}) .{ }^{13} \mathrm{C} \mathrm{NMR}\left(75 \mathrm{MHz}, \mathrm{CDCl}_{3}\right): \delta 179.47,169.65,163.09$, $160.27,156.32$, 133.18, 131.99, 131.43, 131.05, 130.13, 129.21, 128.77, 128.26, 126.02, 122.03, 118.69, 113.99, 111.24, 55.43. HRMS (ESI) calcd for $\mathrm{C}_{25} \mathrm{H}_{20} \mathrm{NO}_{4}$ 
$(\mathrm{M}+\mathrm{H})^{+}:$398.1387, found 398.1395.

Procedure for the synthesis of compound 11.<smiles>CCOC(=O)C(CC)C(=O)/C=C(\O)c1ccccc1</smiles>

To a $10 \mathrm{~mL}$ round-bottom flask was charged with $\mathbf{3 a}(26 \mathrm{mg}, 0.1 \mathrm{mmol}), 10 \mathrm{mg}$ of $\mathrm{NaBH}_{4}(0.25 \mathrm{mmol})$ and $3 \mathrm{~mL}$ of EtOH. The mixture was stirred at room temperature for $4 \mathrm{~h}$. The mixture was concentrated and the residue was purified by chromatography on silica gel using hexane/EtOAc $(1: 1)$ as the eluent to afford $25 \mathrm{mg}$ of compound $\mathbf{1 1}$. Ethyl 2-ethyl-5-hydroxy-3-oxo-5-phenylpent-4-enoate (11). Colorless liquid. ${ }^{1} \mathrm{H}$<smiles>CCOC(=O)C(CC)C(=O)/C=C(\O)c1ccccc1</smiles>
NMR (300 MHz, $\left.\mathrm{CDCl}_{3}\right): \delta 15.85$ (brs, $\left.1 \mathrm{H}\right), 7.88(\mathrm{~d}, J=7.2 \mathrm{~Hz}$ $2 \mathrm{H}), 7.56-7.42(\mathrm{~m}, 3 \mathrm{H}), 6.28(\mathrm{~s}, 1 \mathrm{H}), 4.21(\mathrm{q}, J=7.1 \mathrm{~Hz}, 2 \mathrm{H})$, $3.31(\mathrm{t}, J=7.5 \mathrm{~Hz}, 1 \mathrm{H}), 2.09-1.90(\mathrm{~m}, 2 \mathrm{H}), 1.28(\mathrm{t}, J=7.2 \mathrm{~Hz}$, 3H), $1.00(\mathrm{t}, J=7.2 \mathrm{~Hz}, 3 \mathrm{H}) .{ }^{13} \mathrm{C} \mathrm{NMR}\left(125 \mathrm{MHz}, \mathrm{CDCl}_{3}\right): \delta 193.41,182.24,170.23$, 134.26, 132.49, 128.66, 127.05, 95.67, 61.29, 57.84, 22.98, 14.13, 11.94. HRMS (ESI) calcd for $\mathrm{C}_{15} \mathrm{H}_{19} \mathrm{O}_{4}(\mathrm{M}+\mathrm{H})^{+}:$263.1278, found 263.1284 .

Procedure for the synthesis of product $3 \mathrm{~g}$ from acid chloride.<smiles>COC(=O)c1c(-c2ccccc2)oc(-c2ccccc2)cc1=O</smiles>

To an oven-dried $25 \mathrm{~mL}$ round-bottom flask was charged with acid $1 \mathbf{a}$ (51 mg, 0.35 mmol) and $5 \mathrm{~mL}$ of $\mathrm{SOCl}_{2}$. The resulting mixture was refluxed for $2 \mathrm{~h}$, and then $\mathrm{SOCl}_{2}$ was removed under reduced pressure. Then, substrate $\mathbf{2 g}$ (125 mg, $0.7 \mathrm{mmol}$ ), DMAP (85 $\mathrm{mg}, 0.7 \mathrm{mmol})$, and $\mathrm{Sc}(\mathrm{OTf})_{3}(30 \mathrm{mg}, 0.06 \mathrm{mmol})$ was added to the residue followed by addition of anhydrous 1,2-DCE (4 mL). The resulting mixture was heated at $80^{\circ} \mathrm{C}$ in air for a period time until the completion of the reaction as monitored by TLC. The mixture was cooled to room temperature. The mixture was filtered and the filtrate was concentrated under reduced pressure. The residue was purified by chromatography on silica gel using hexane/EtOAc (10:1) as the eluent to afford 56 
$\mathrm{mg}$ of product $\mathbf{3 g}(52 \%)$.

Procedure for the reaction of alkynyl acyl imidazole 12 with $2 \mathrm{~g}$.<smiles></smiles>

To an oven-dried $10 \mathrm{~mL}$ round-bottom flask was charged with 12 (39 mg, $0.2 \mathrm{mmol}$ ) $2 \mathrm{~g}$ (71 mg, $0.4 \mathrm{mmol}$ ), additive (none or certain amount of DMAP), $\mathrm{Sc}(\mathrm{OTf})_{3}(17 \mathrm{mg}$, $0.034 \mathrm{mmol}), 200 \mathrm{mg}$ of $4 \AA \mathrm{MS}$ and $\mathrm{NaOH}(10 \mathrm{mg}, 0.24 \mathrm{mmol})$. Then anhydrous 1,2-DCE ( $3 \mathrm{~mL}$ ) was added to the flask and the resulting mixture was heated at $80{ }^{\circ} \mathrm{C}$ in air for a period time until the completion of the reaction as monitored by TLC. The mixture was cooled to room temperature. The mixture was concentrated under reduced pressure. The residue was purified by chromatography on silica gel using hexane/EtOAc (10:1) as the eluent to afford products $\mathbf{3 g}$ and/or $\mathbf{4 g}$. $6 \mathrm{mg}$ of $\mathbf{3 g}$ and 31 $\mathrm{mg}$ of $\mathbf{4 g}$ were obtained when none of additive was added. $32 \mathrm{mg}$ of $\mathbf{3 g}$ and $5 \mathrm{mg}$ of $\mathbf{4 g}$ were obtained when $20 \mathrm{~mol} \%$ of DMAP was added. $40 \mathrm{mg}$ of $\mathbf{3 g}$ was obtained when 1.1 equiv of DMAP was added.<smiles>COc1c(-c2ccccc2)cc(=O)oc1-c1ccccc1</smiles>

Methyl 2-oxo-4,6-diphenyl-2H-pyran-5-carboxylate

$(4 \mathrm{~g})$.

Colorless liquid. Known compound. ${ }^{[5]}{ }^{1} \mathrm{H}$ NMR (300 MHz, $\mathrm{CDCl}_{3}$ ): $\delta 7.64(\mathrm{~d}, J=6.6 \mathrm{~Hz}, 2 \mathrm{H}), 7.50-7.43(\mathrm{~m}, 6 \mathrm{H}), 7.37-7.35(\mathrm{~m}, 2 \mathrm{H})$, $4 \mathrm{~g} \mathrm{CO} \mathrm{CO}_{2} \mathrm{Me} 6.29(\mathrm{~s}, 1 \mathrm{H}), 3.46(\mathrm{~s}, 3 \mathrm{H})$.

\section{References:}

[1] a) J. Lim, J. Choi, H.-S. Kim, I. S. Kim, K. C. Nam, J. Kim, S. Lee, J. Org. Chem. 2016, 81, 303; b) D. R. Stuart, P. Alsabeh, M. Kuhn, K. Fagnou, J. Am. Chem. Soc. 2010, 132, 18326.

[2] R. Balamurugan, S. Manojveer, Chem. Commun. 2011, 47, 11143.

[3] N. Latif, E. T. Kaiser, J. Org. Chem. 1969, 34, 3653.

[4] D. Schmidt, J. Conrad, I. Klaiber, U. Beifuss, Chem. Commun. 2006, 4732.

[5] R. Manikandan, M. Jeganmohan Org. Lett. 2014, 16, 652. 

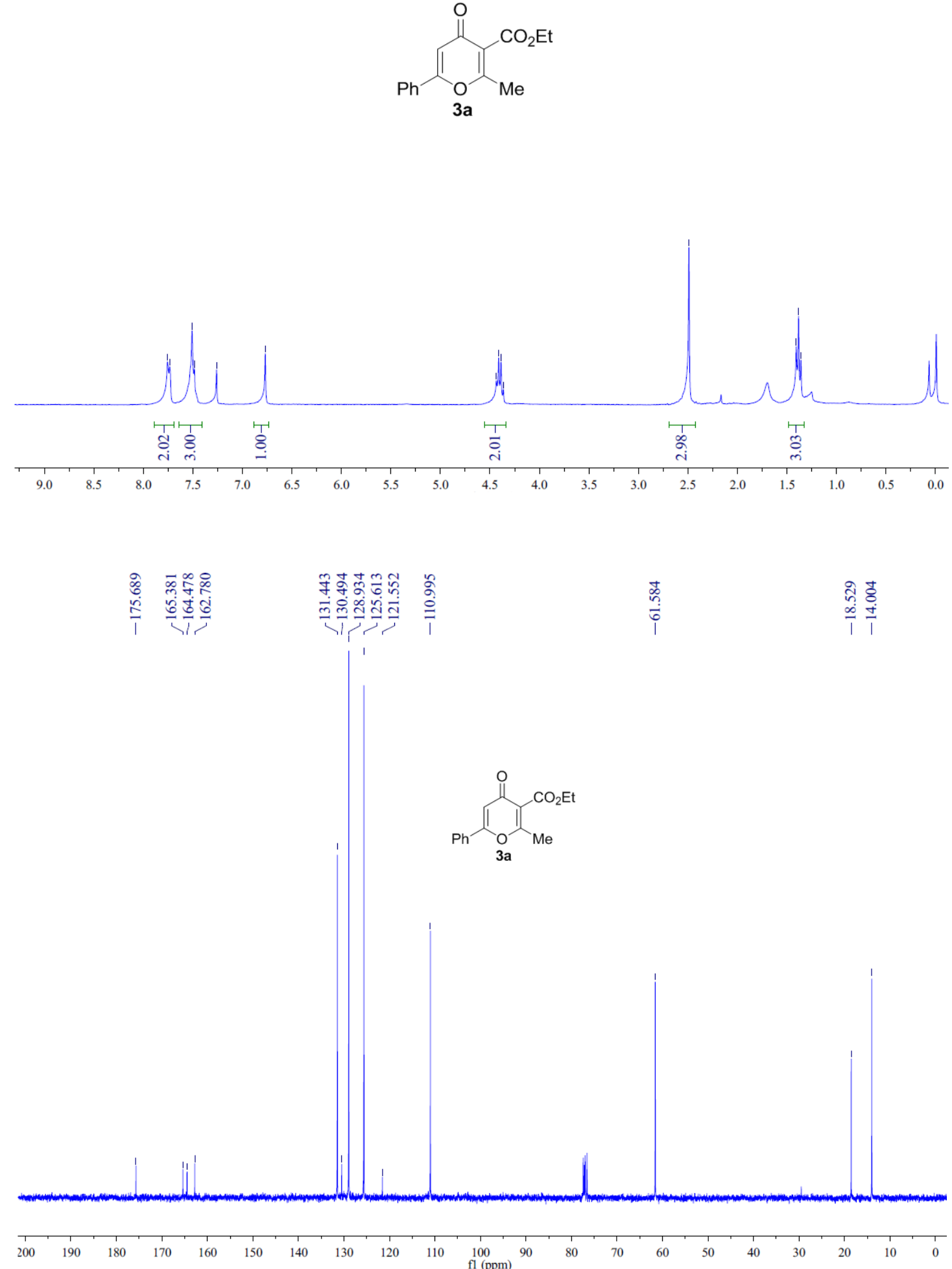


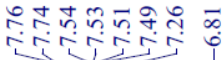

$\stackrel{5}{1} \quad \overline{5}$
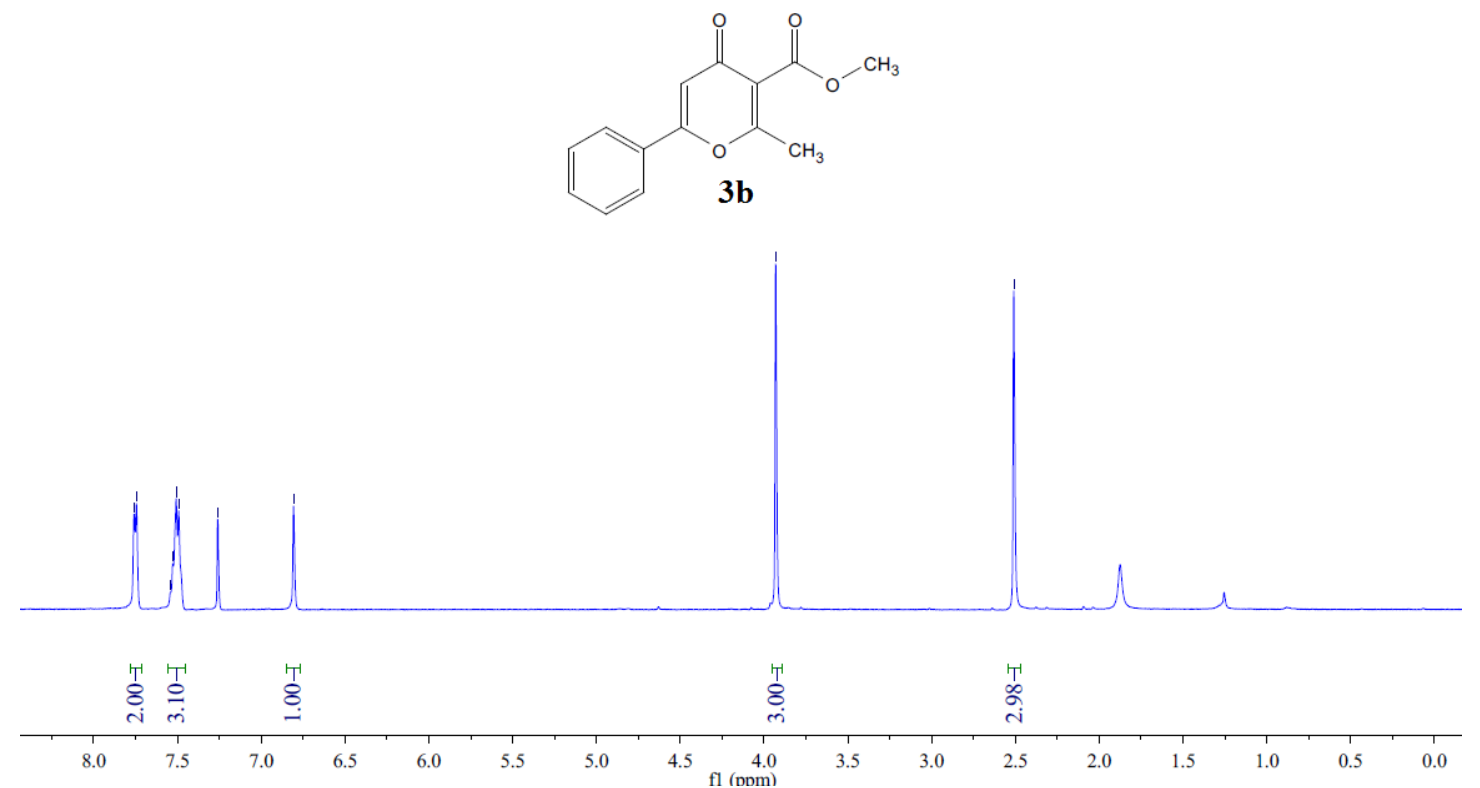

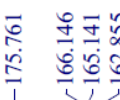

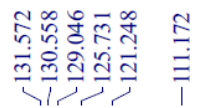

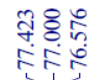

in

$\stackrel{2}{1}$
$\substack{1 \\ \infty}$

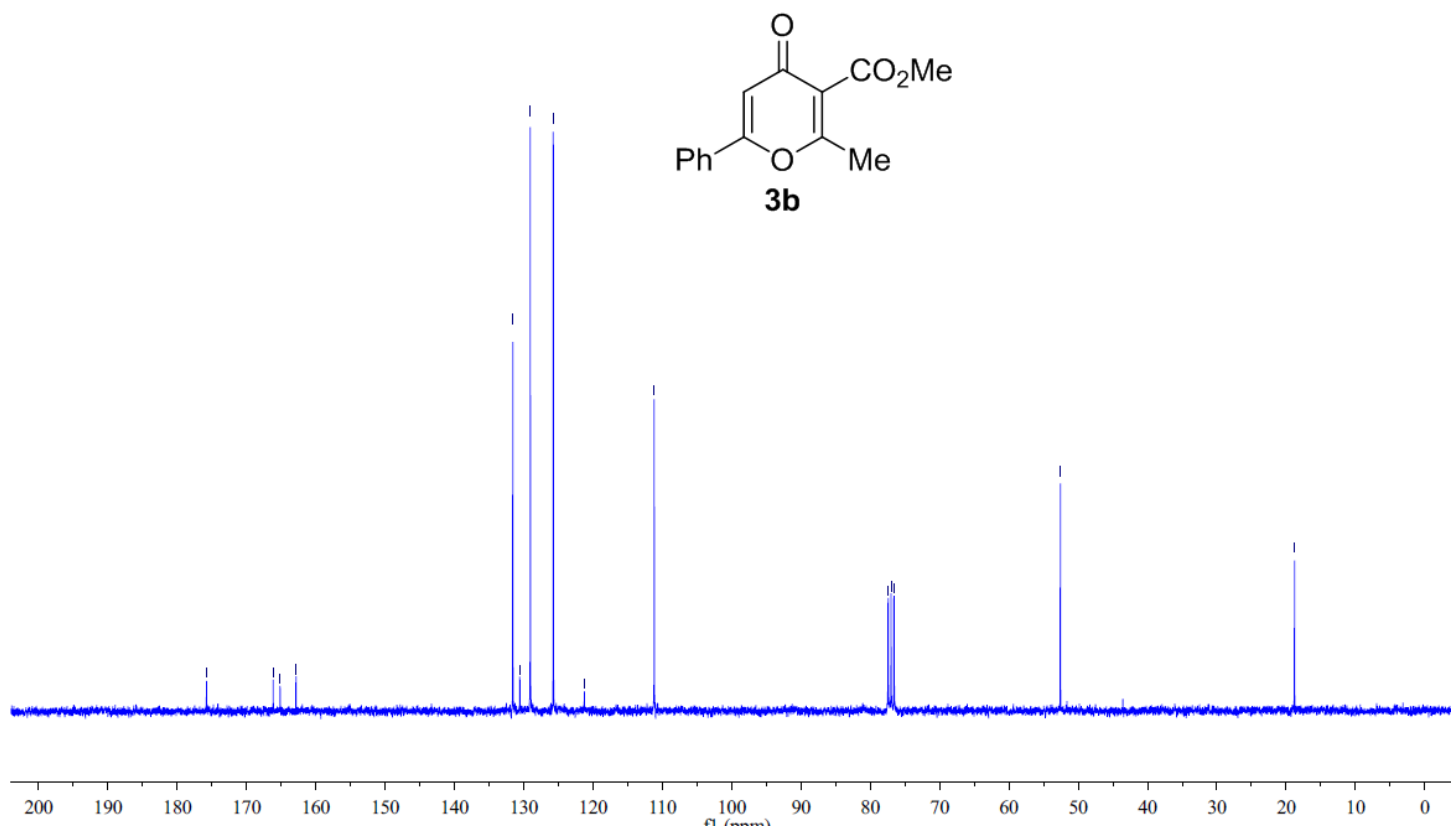




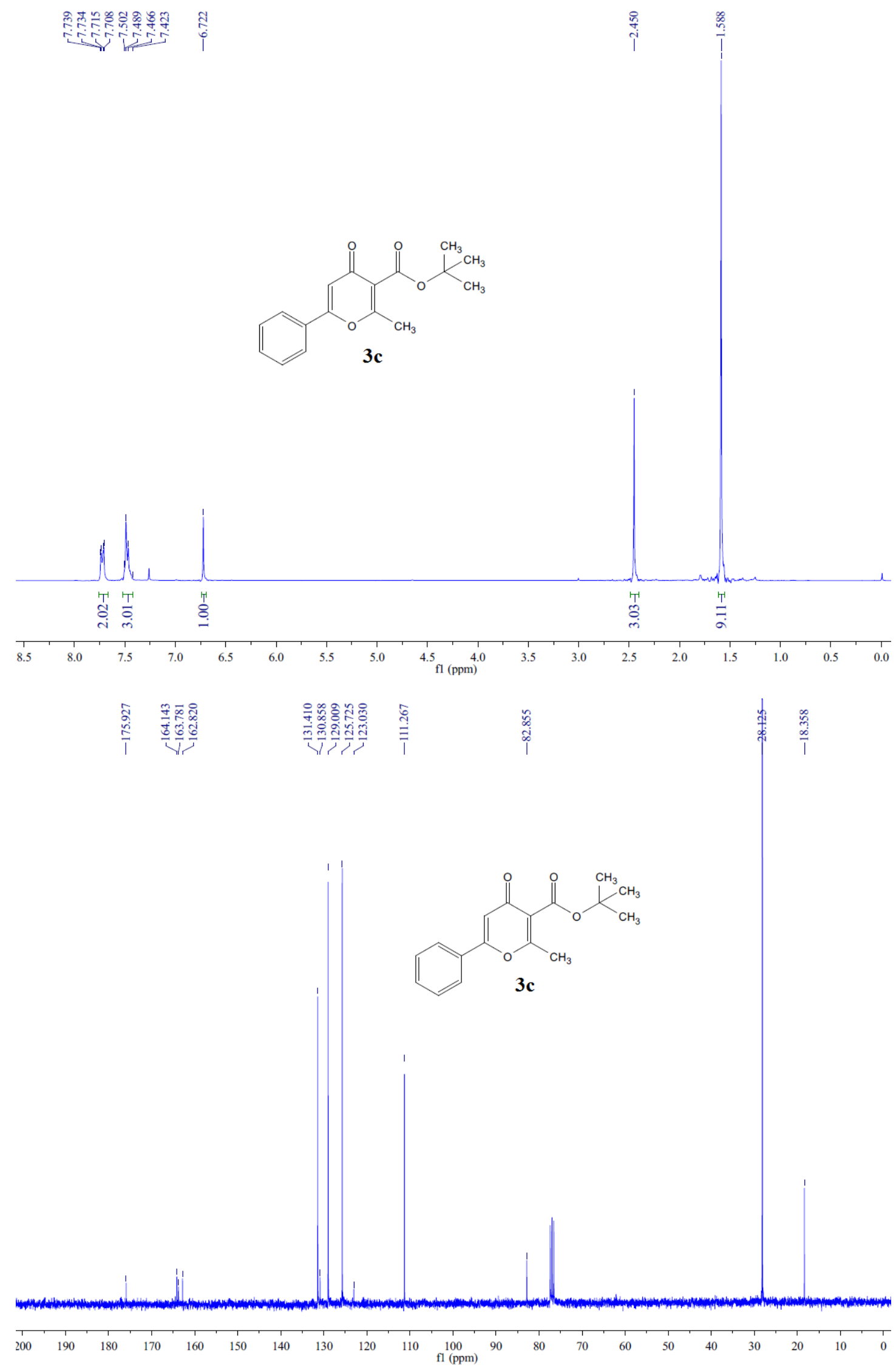


<smiles>Cc1oc(-c2ccccc2)cc(=O)c1C(=O)OCc1ccccc1</smiles>
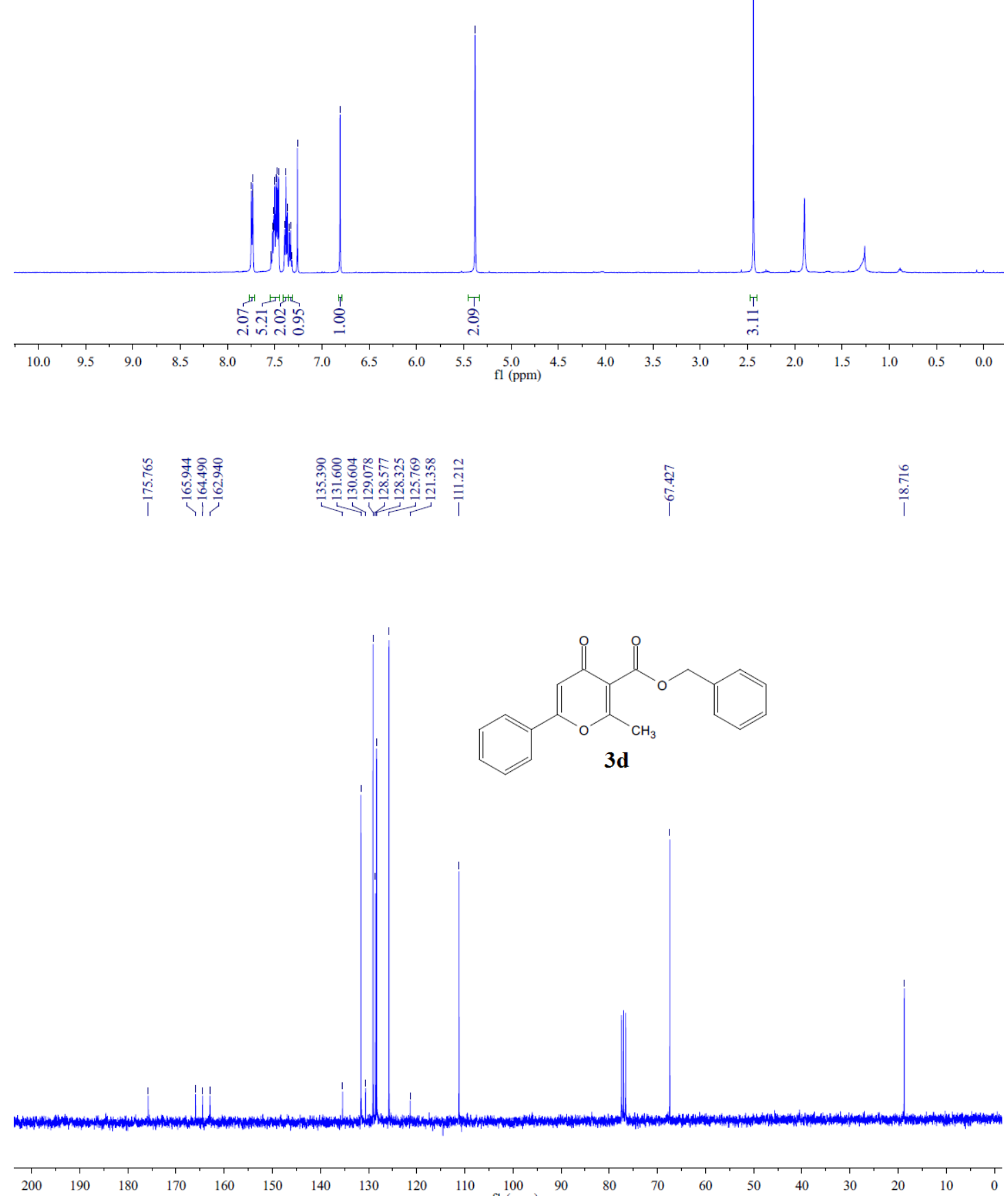

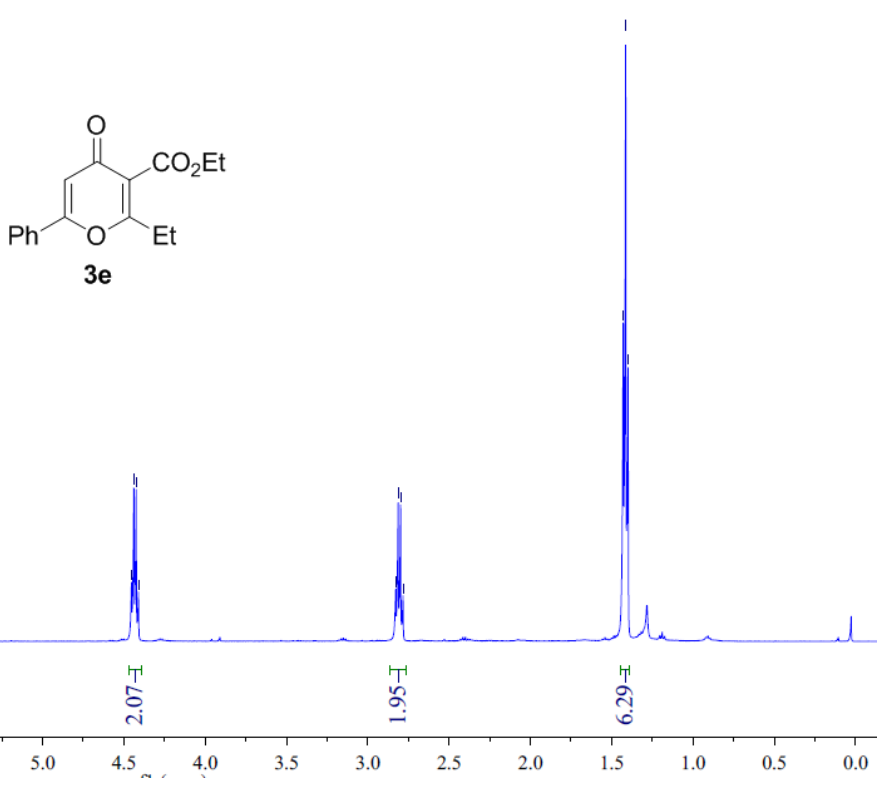

ริ도웡

은

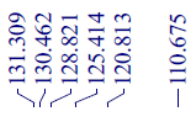

京

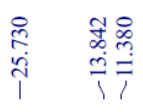

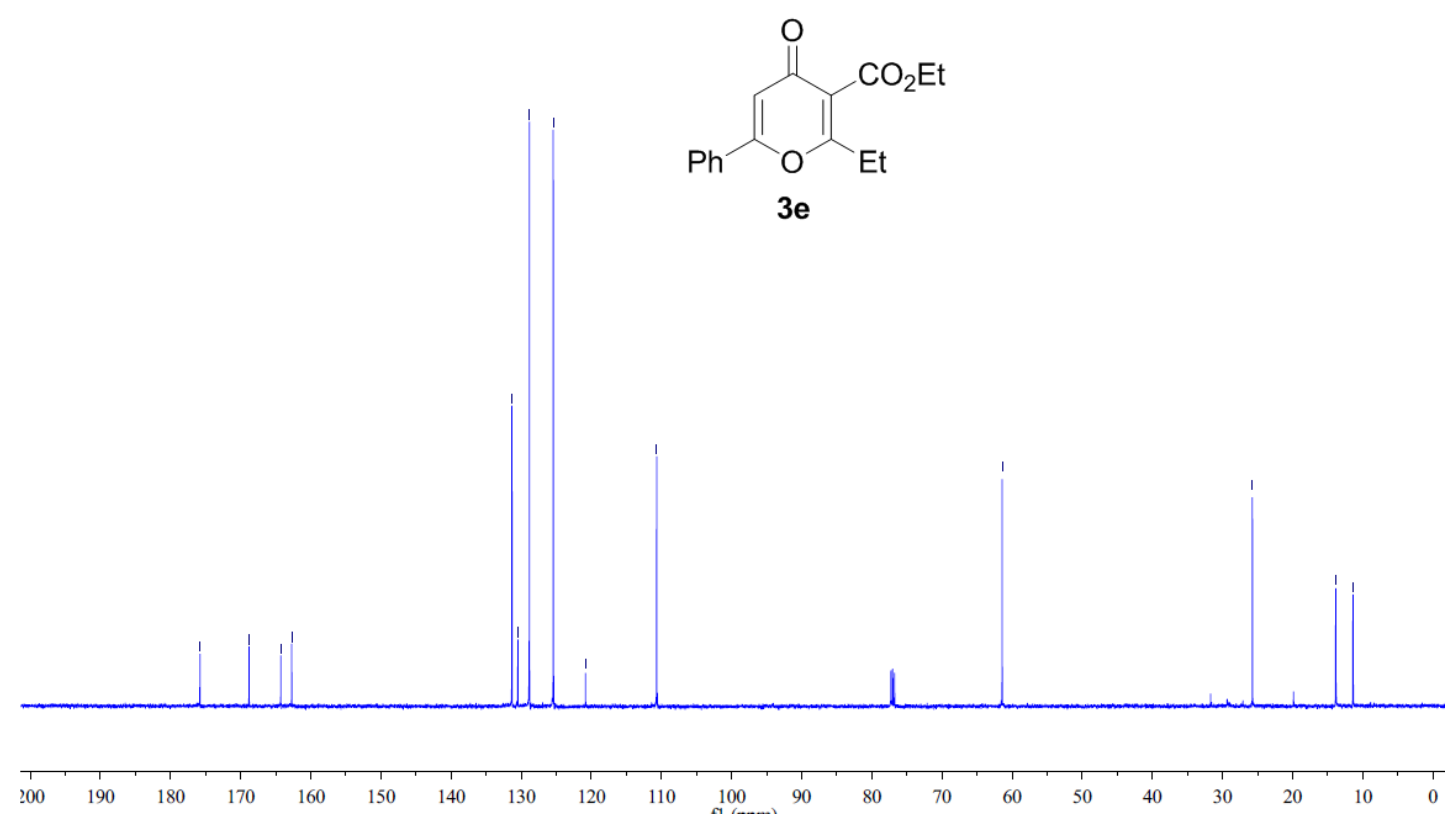



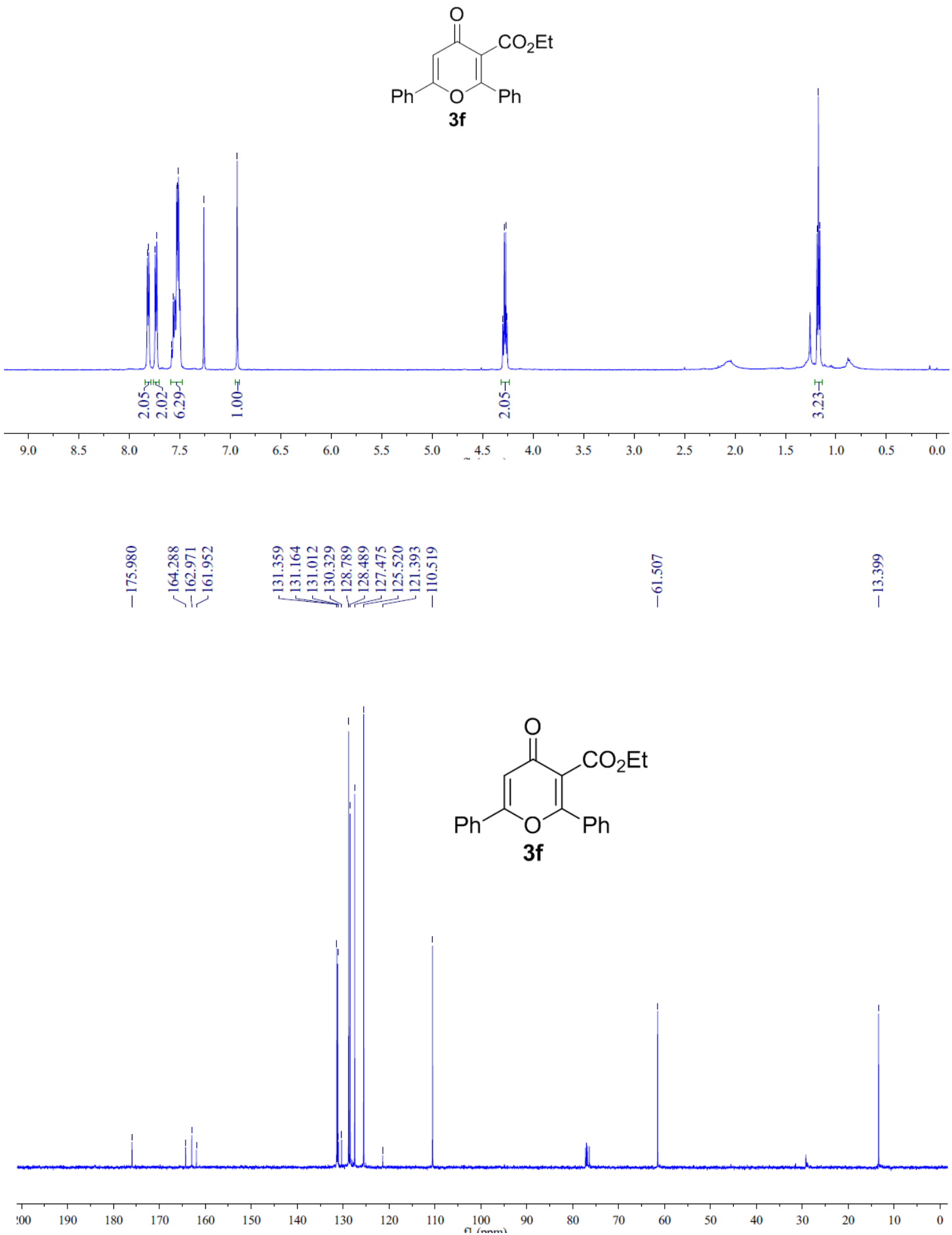


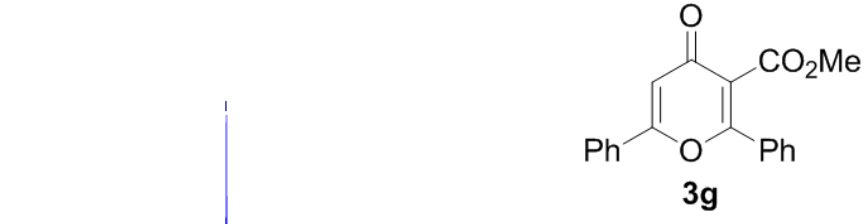

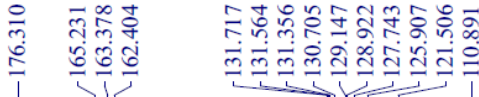

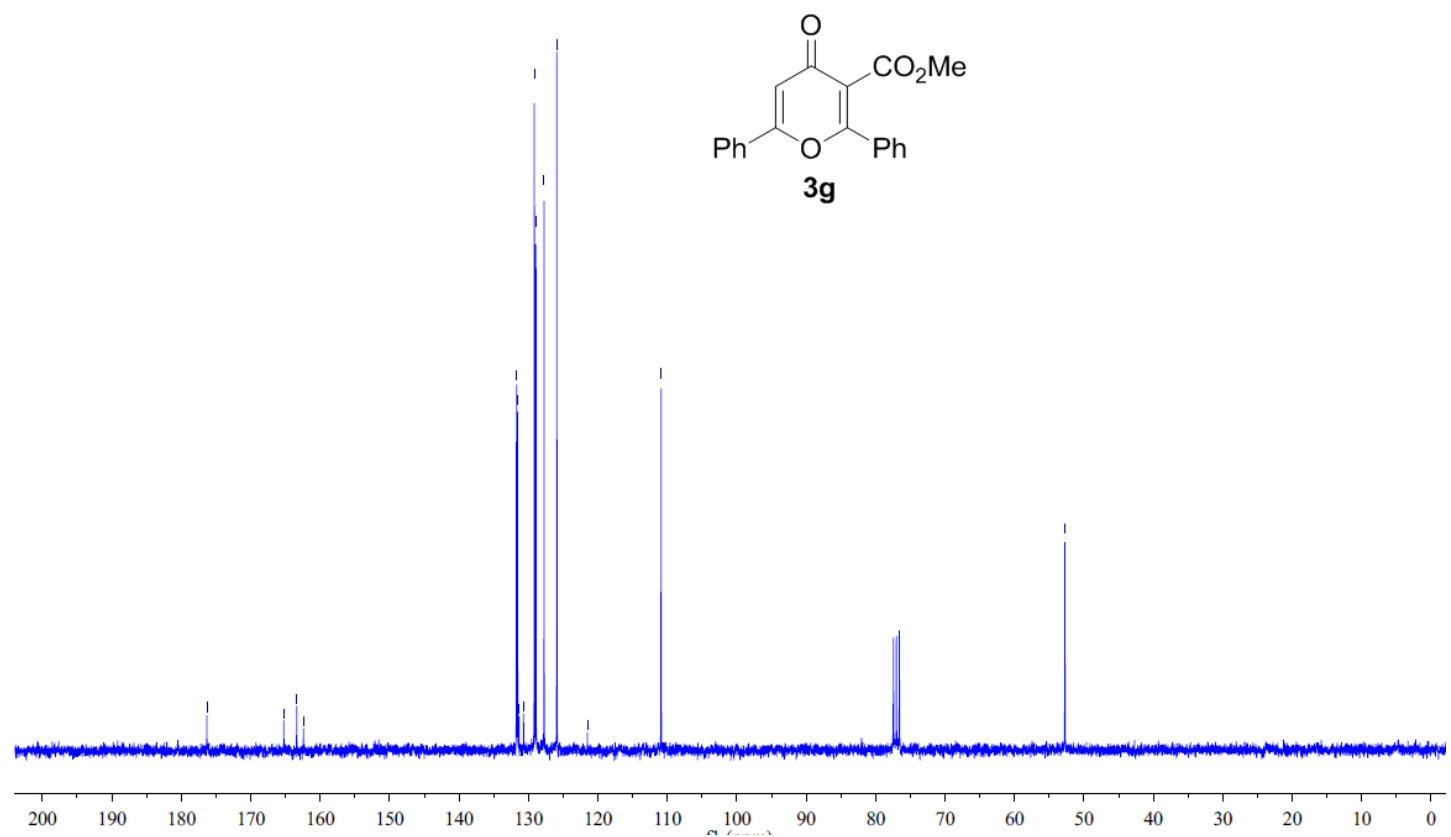



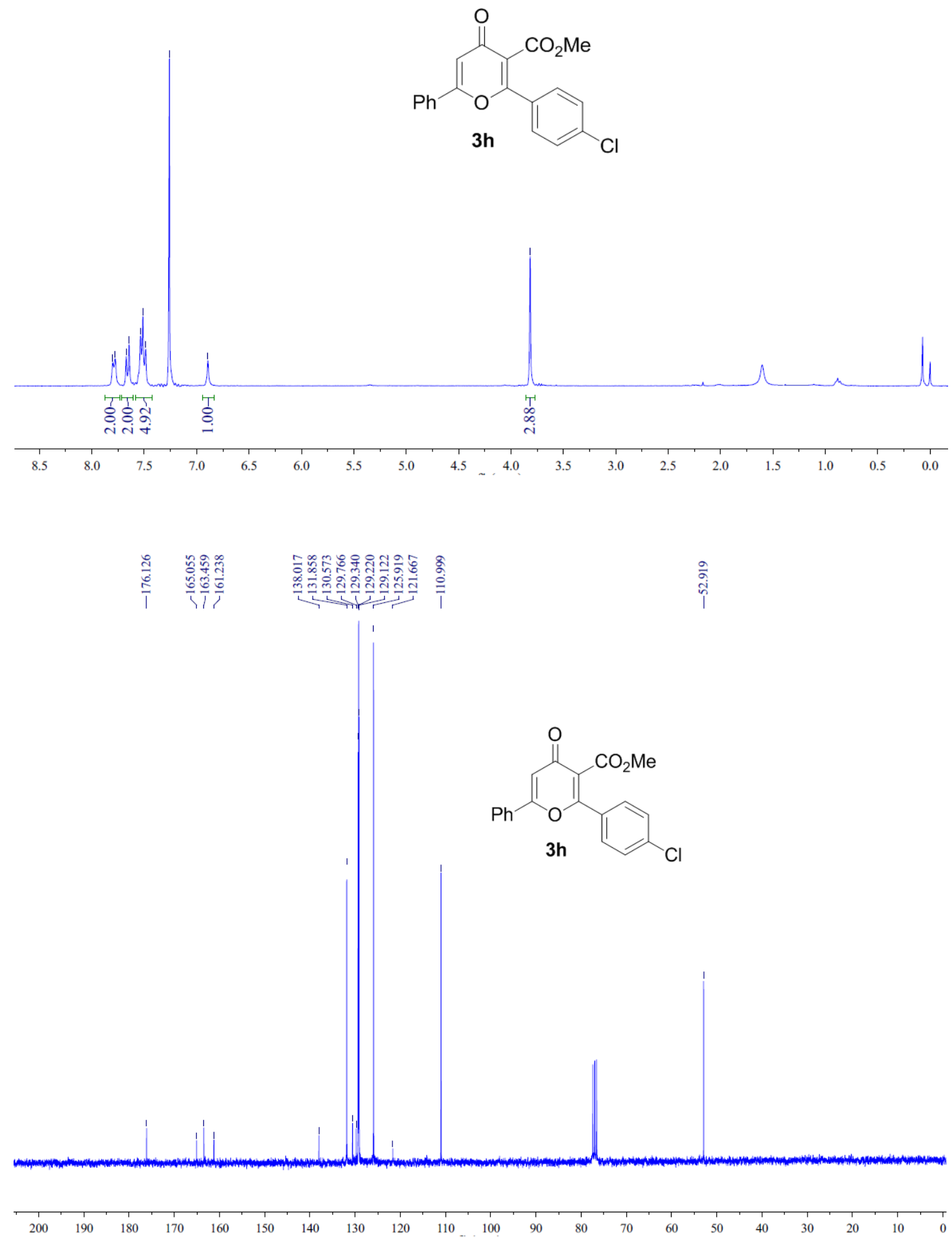


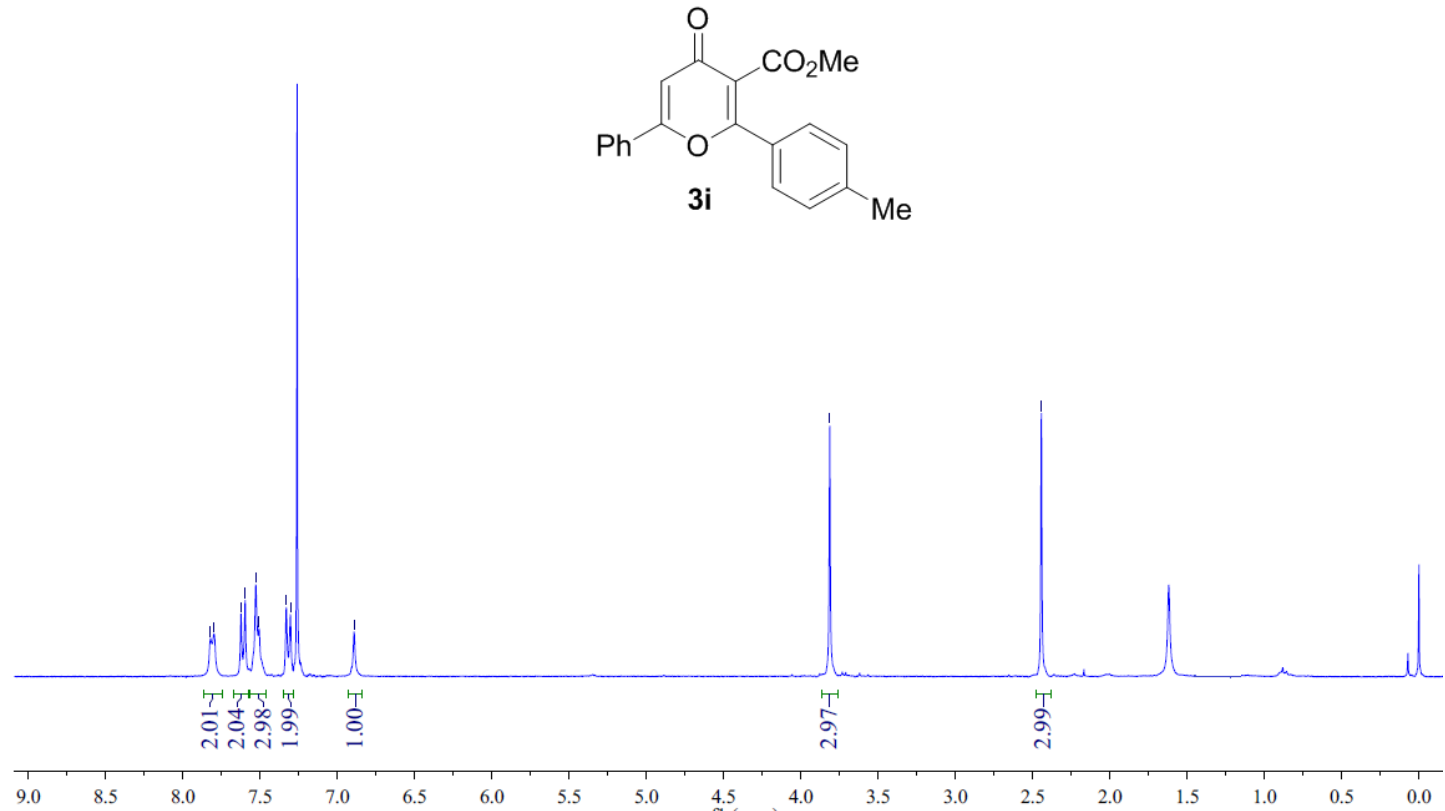

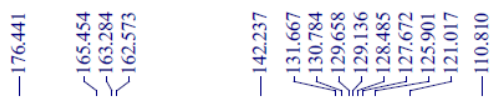
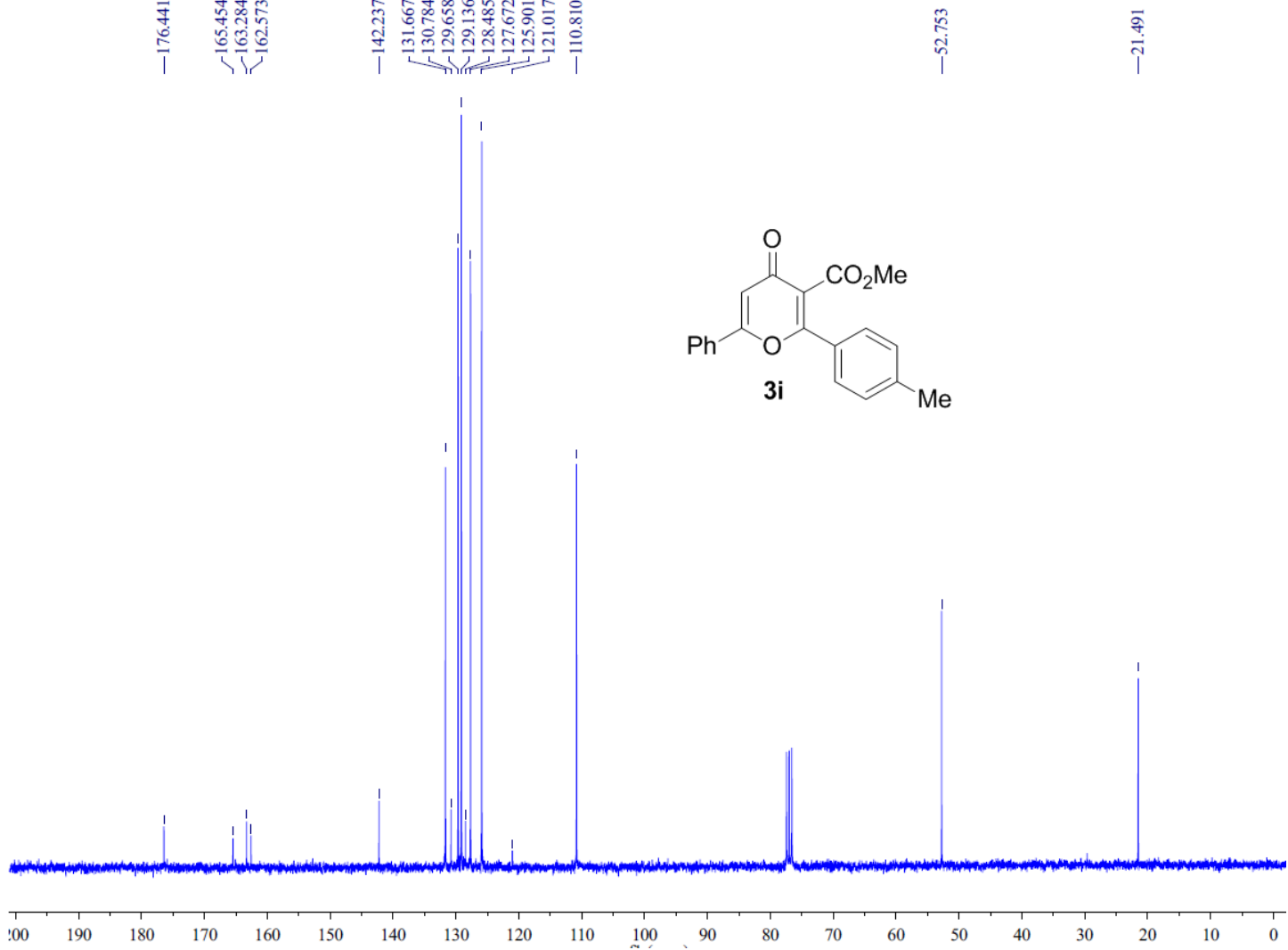


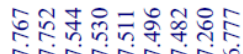

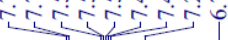

งั้ำ
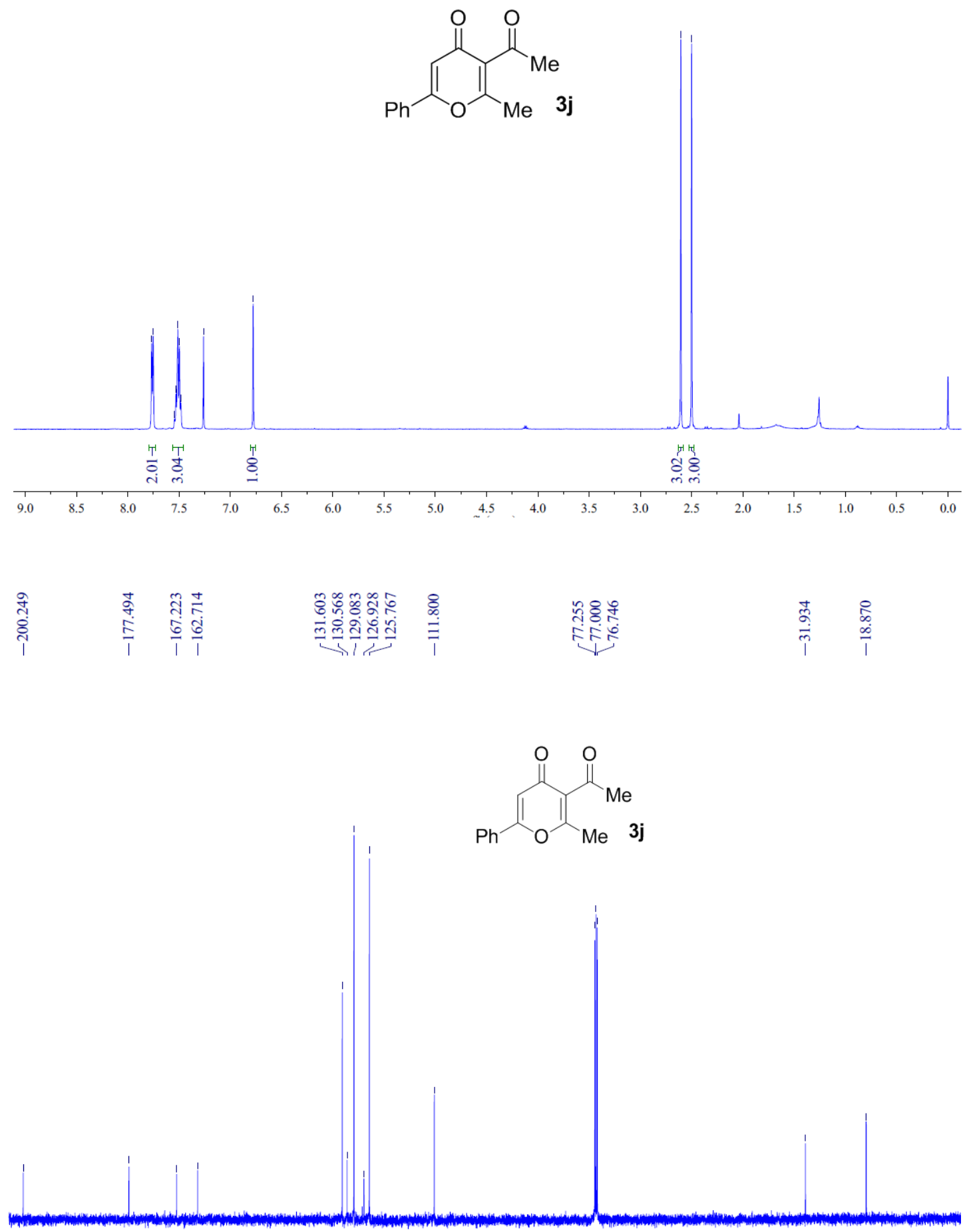

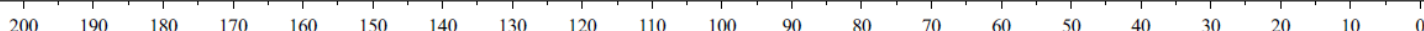



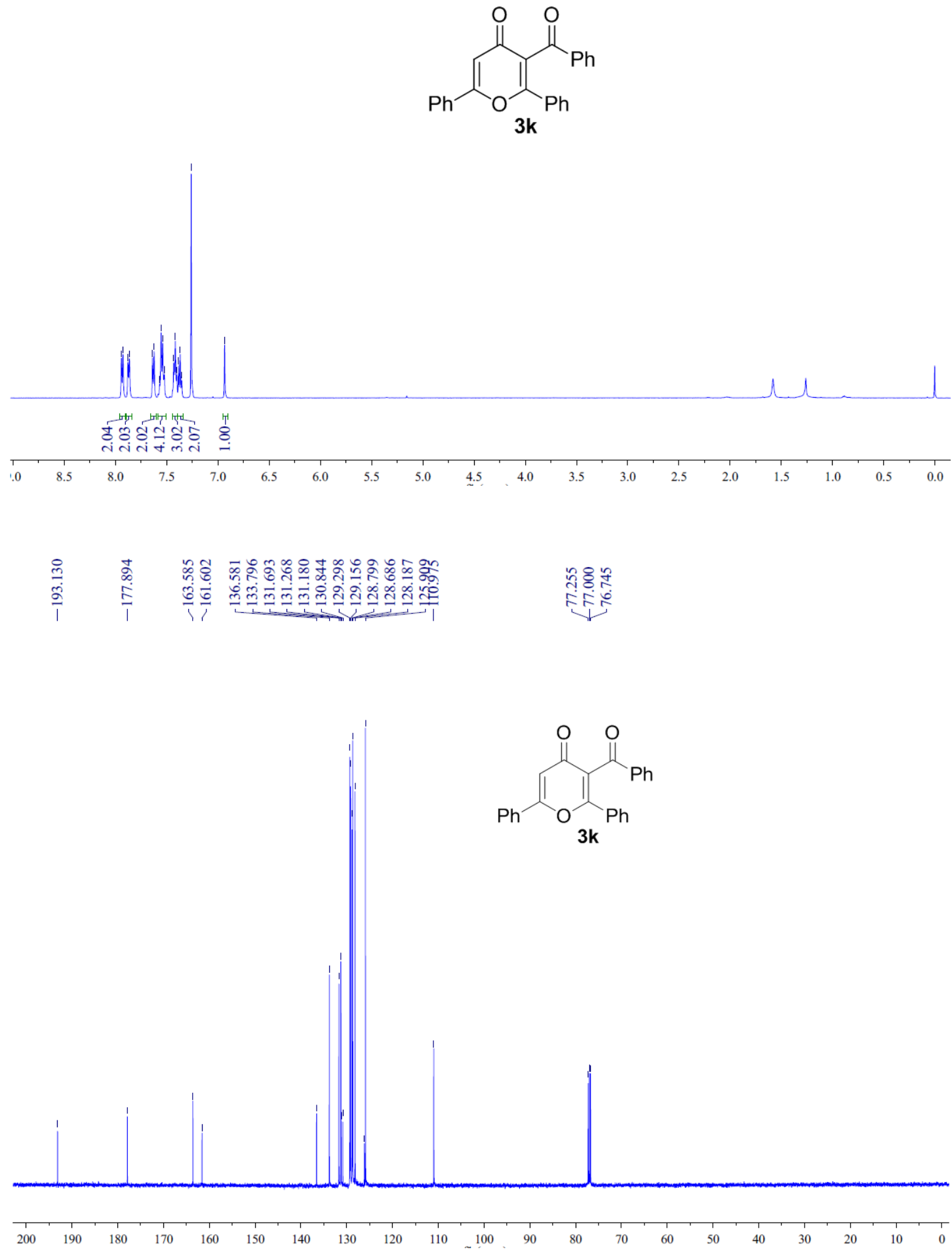

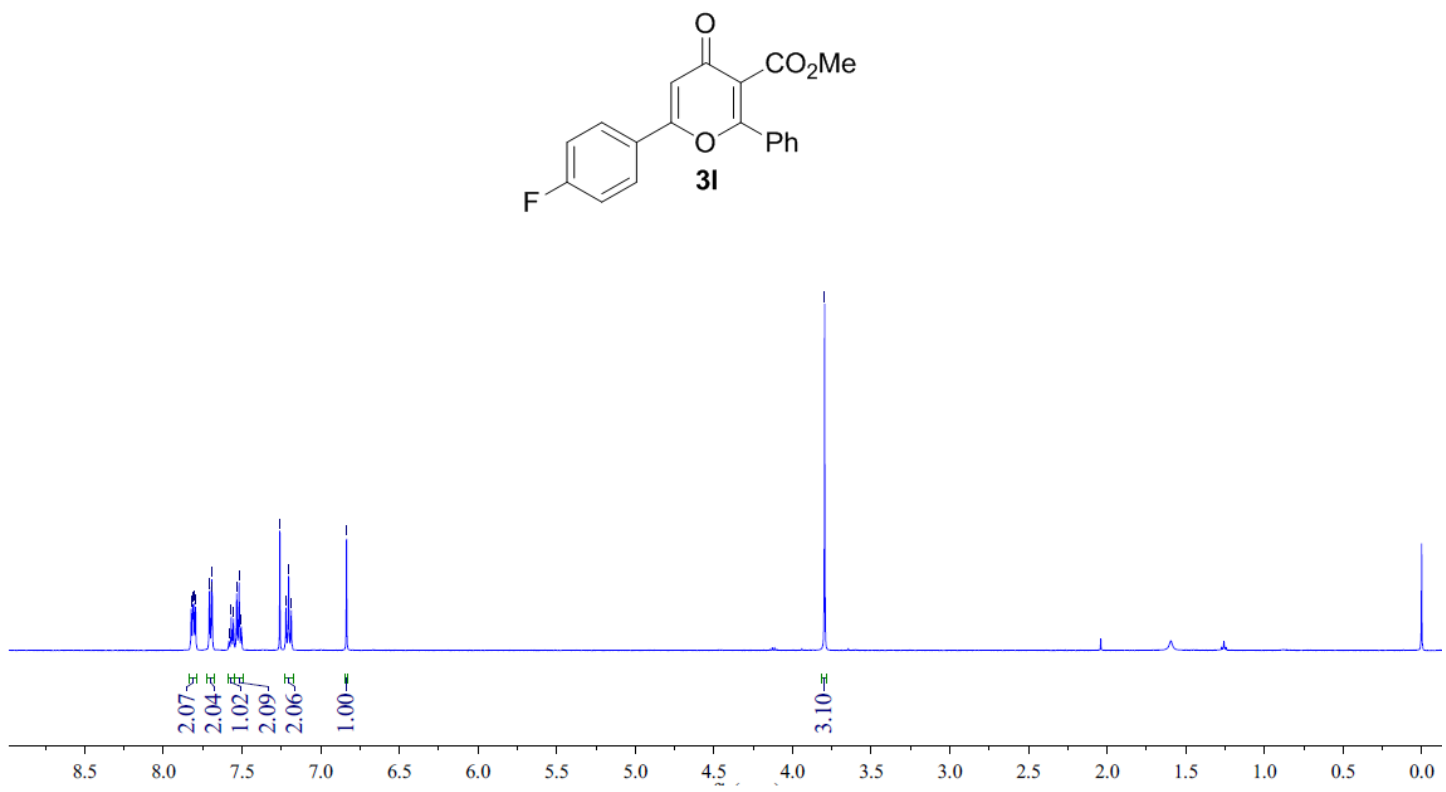

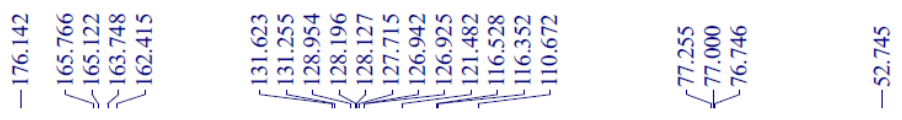

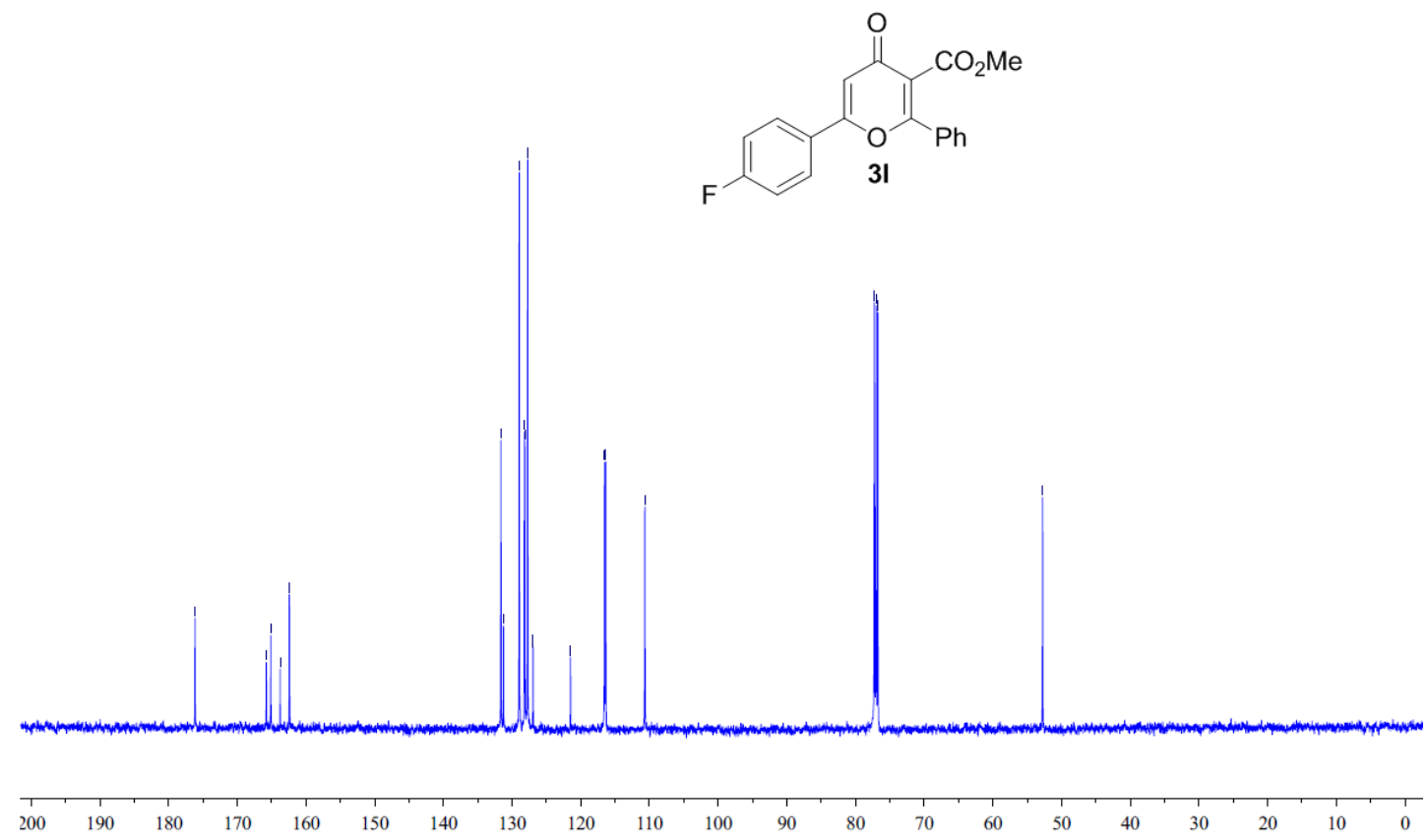




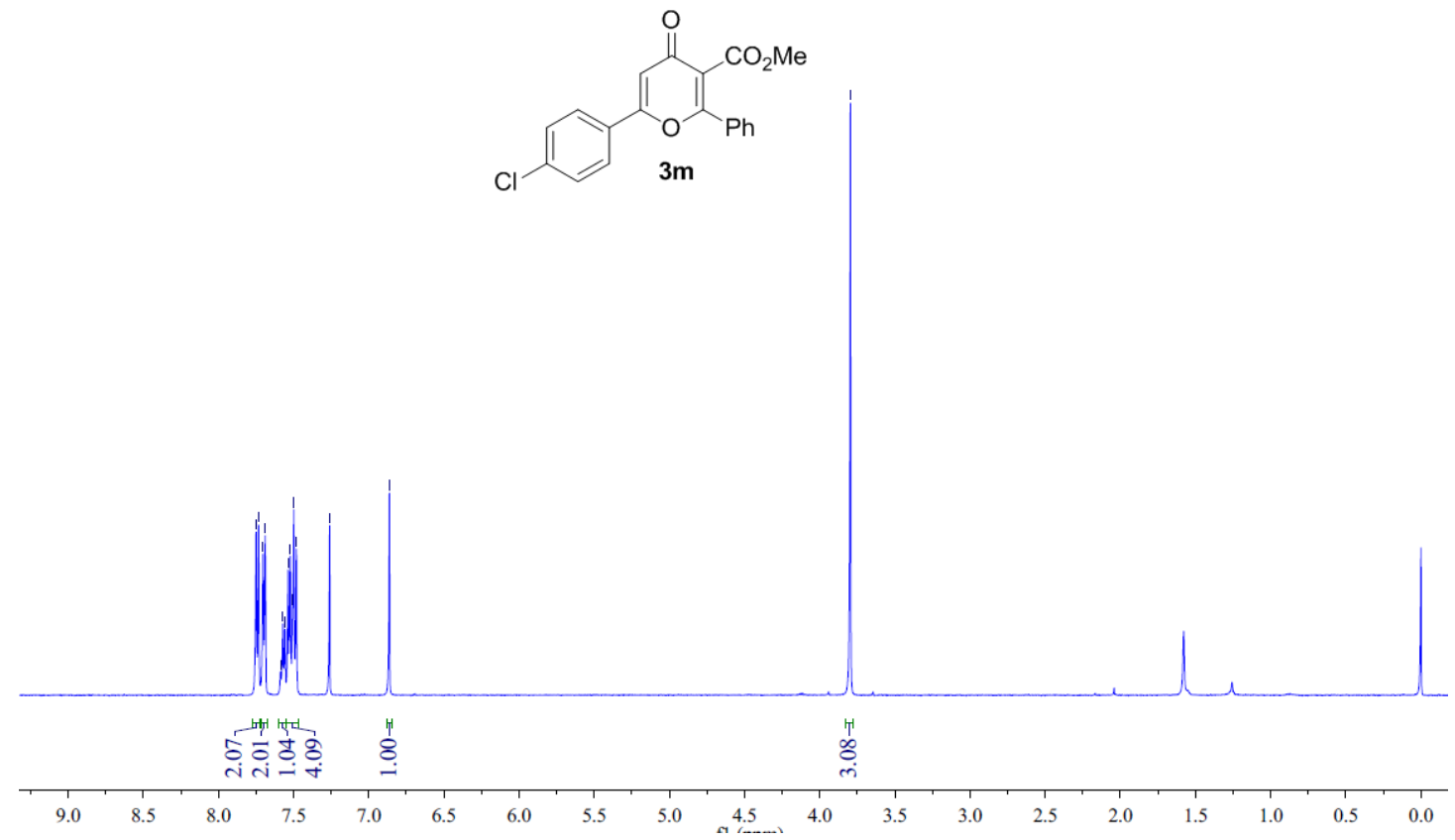

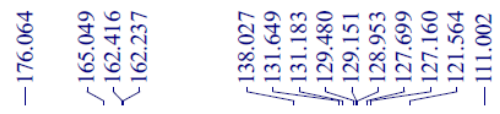

तุํํㅇำ

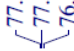
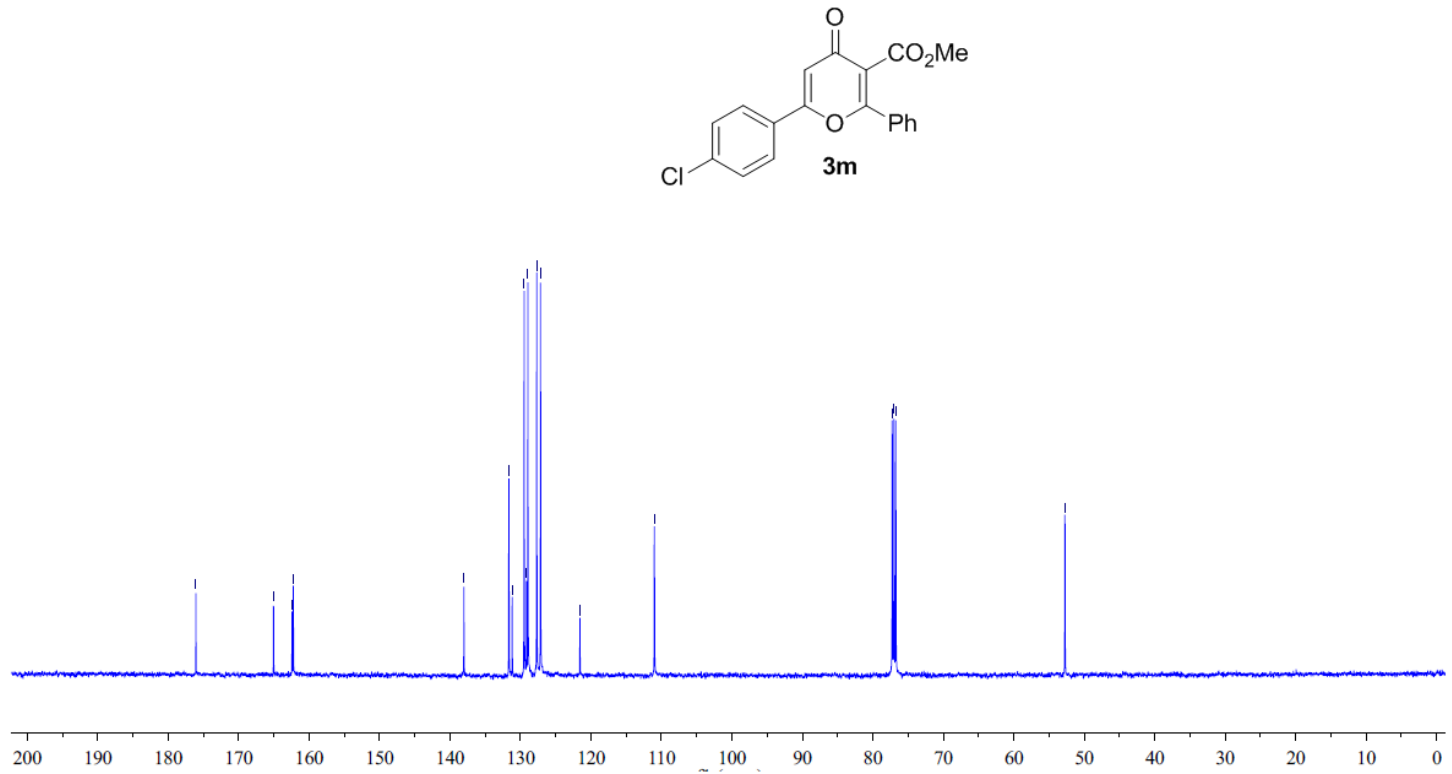

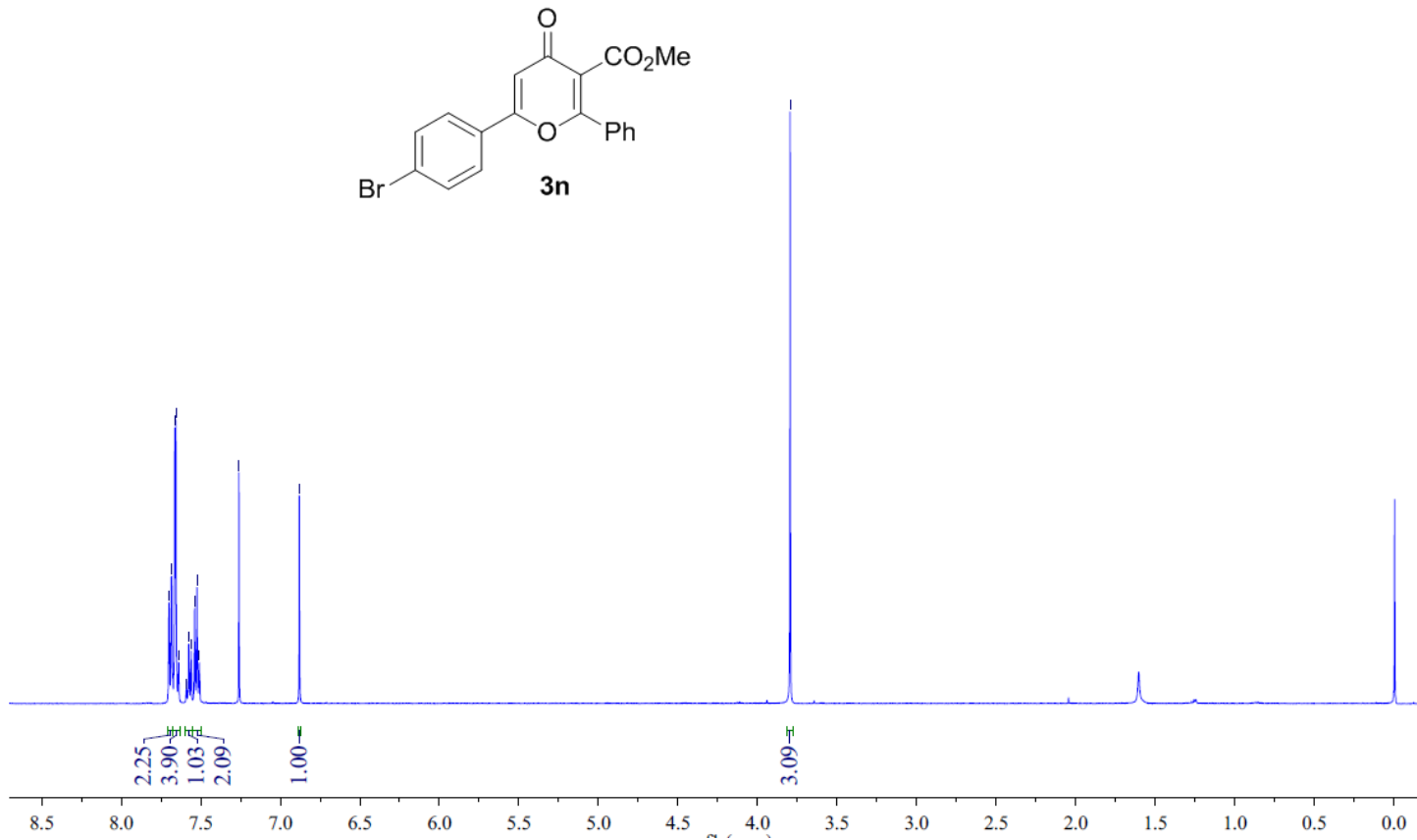

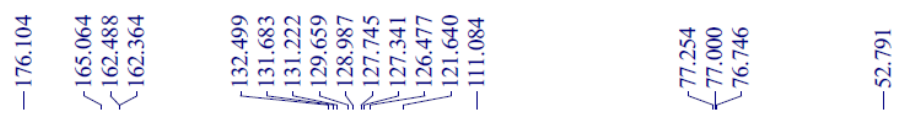

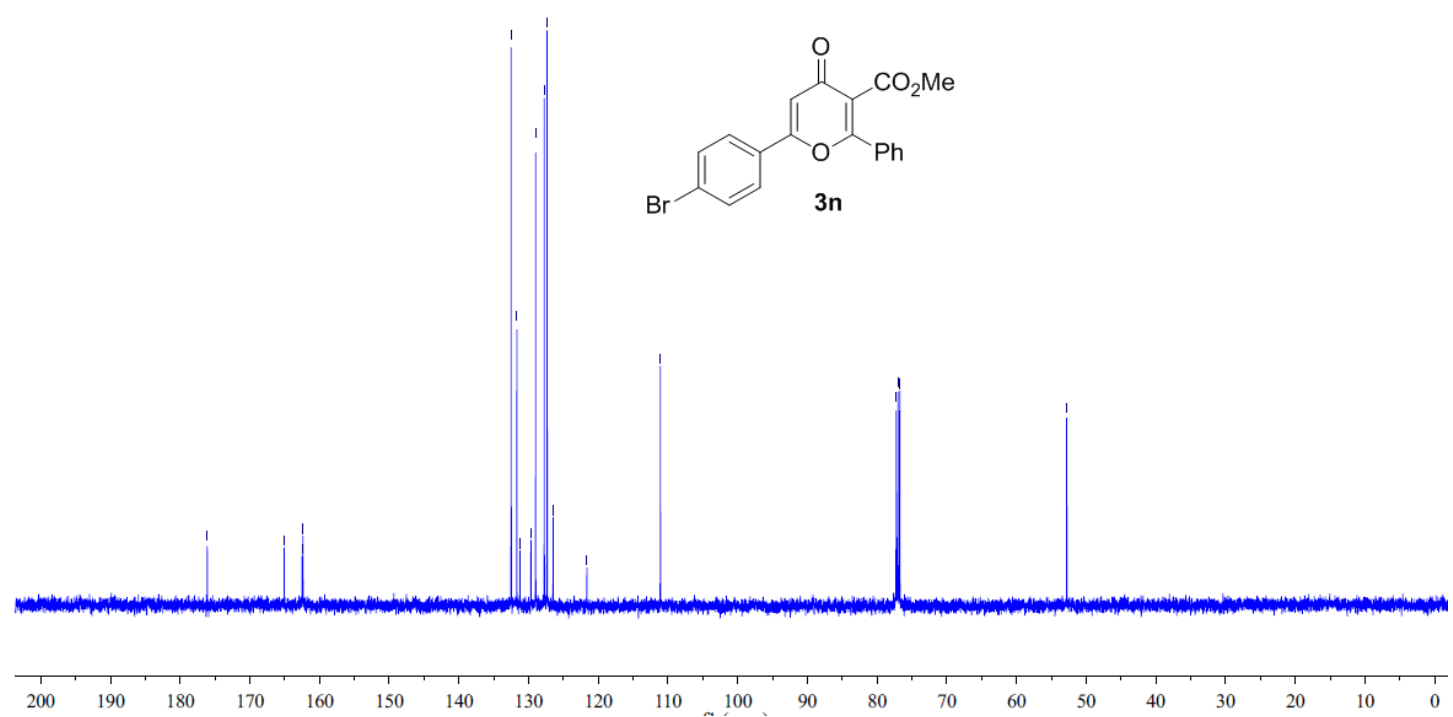




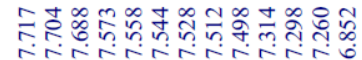

resing

$\stackrel{\substack{1 \\ \infty}}{\stackrel{8}{7}}$
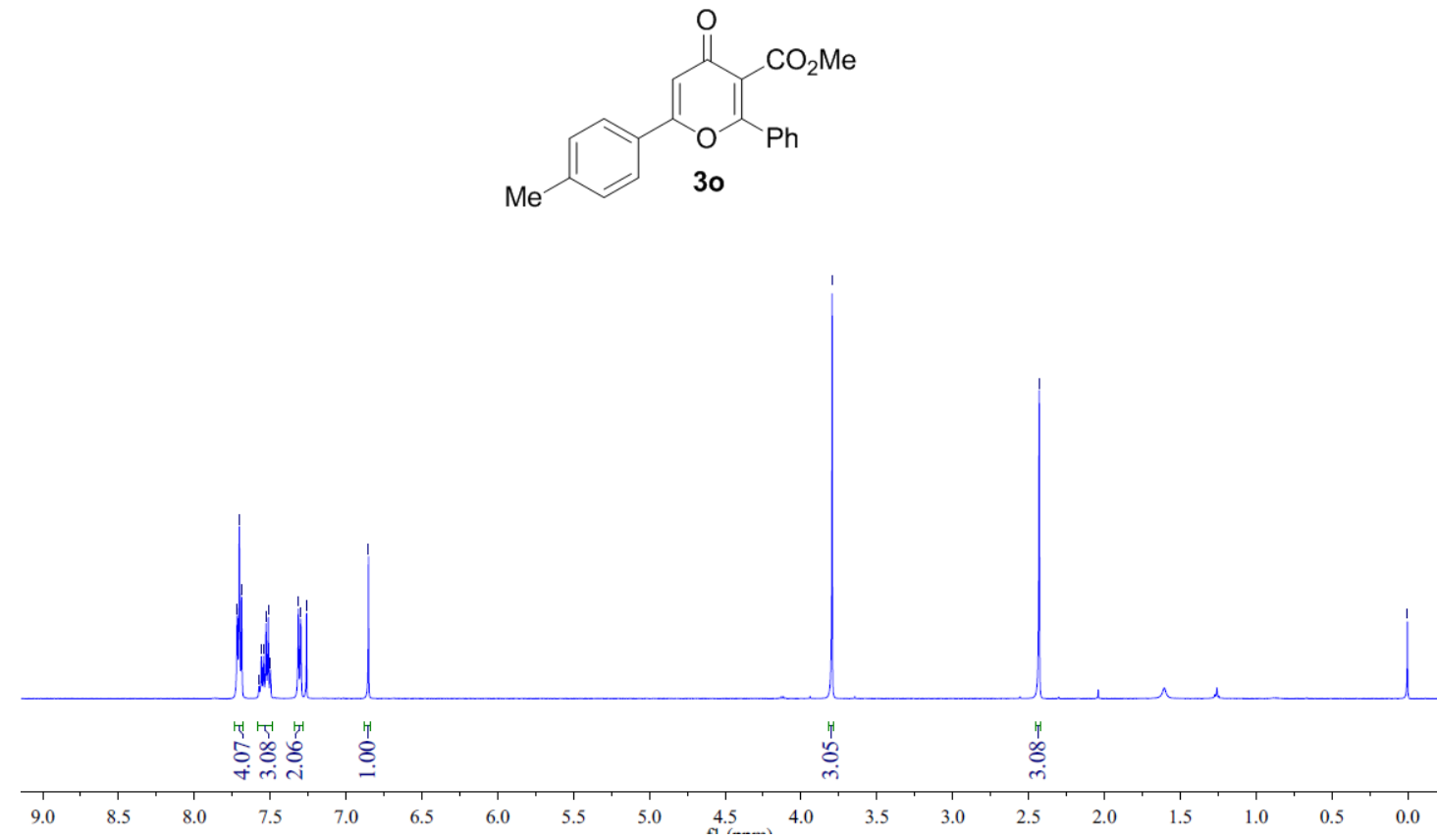

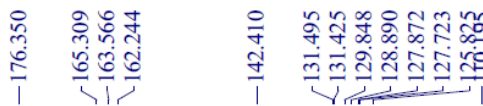

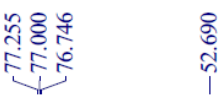

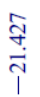
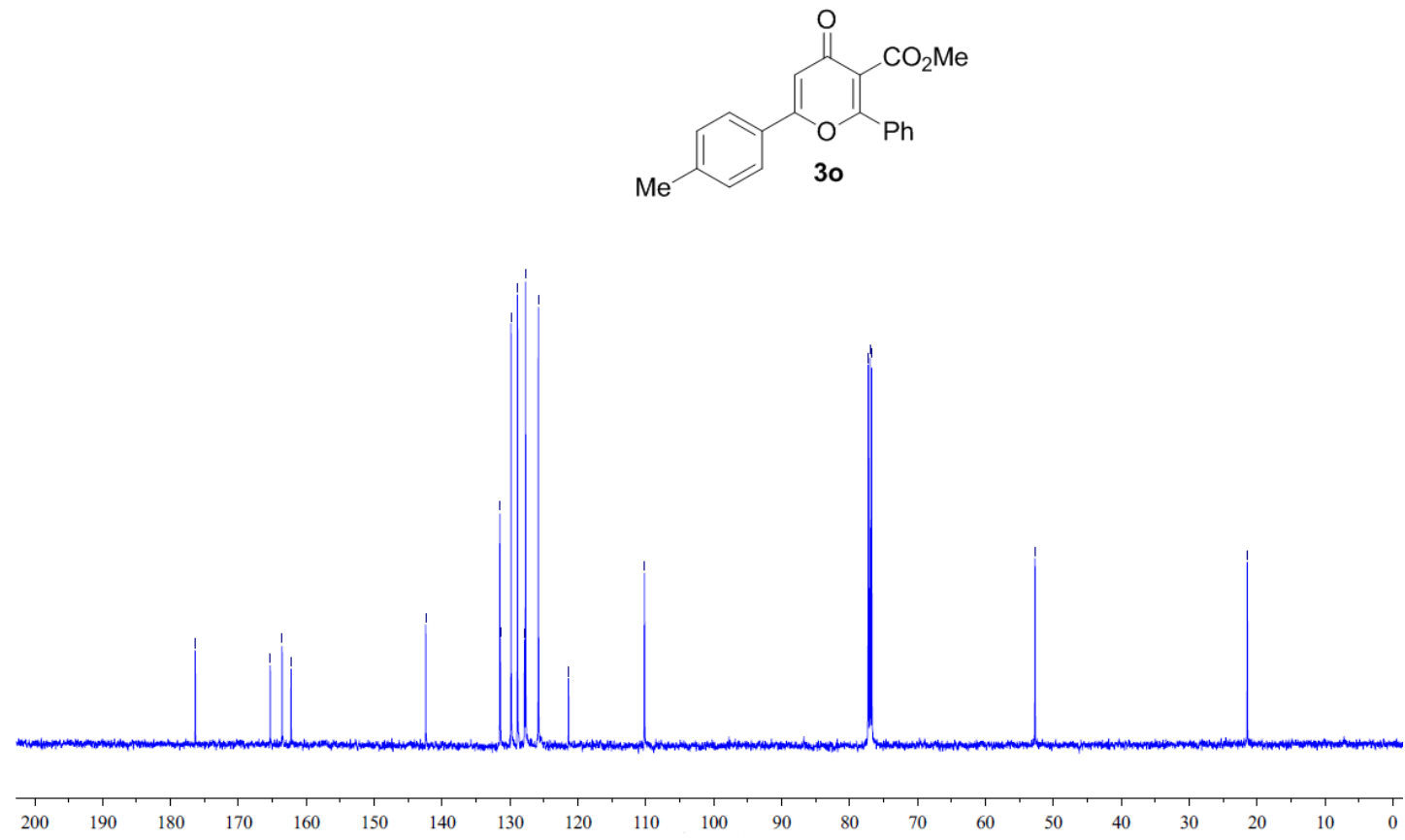

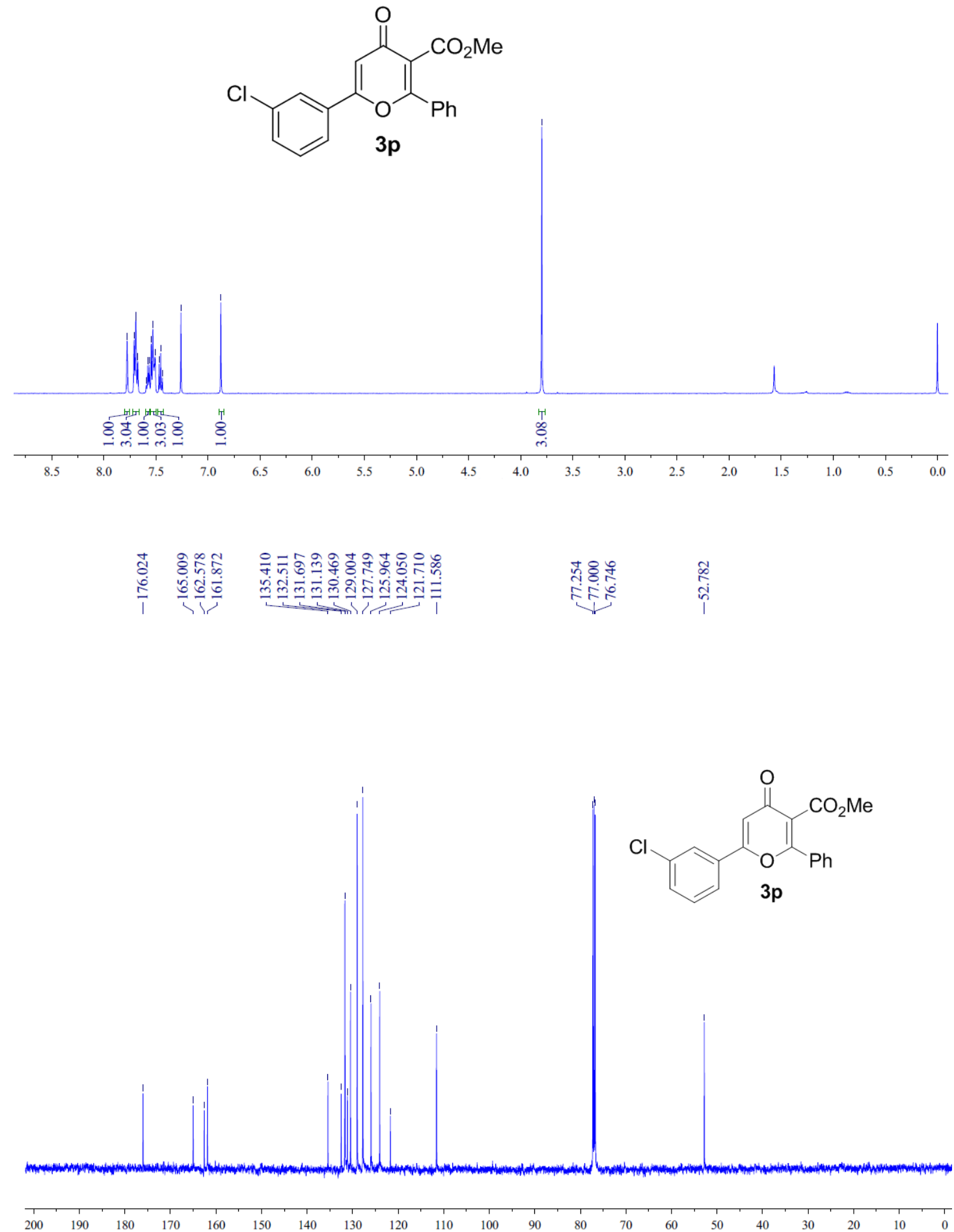


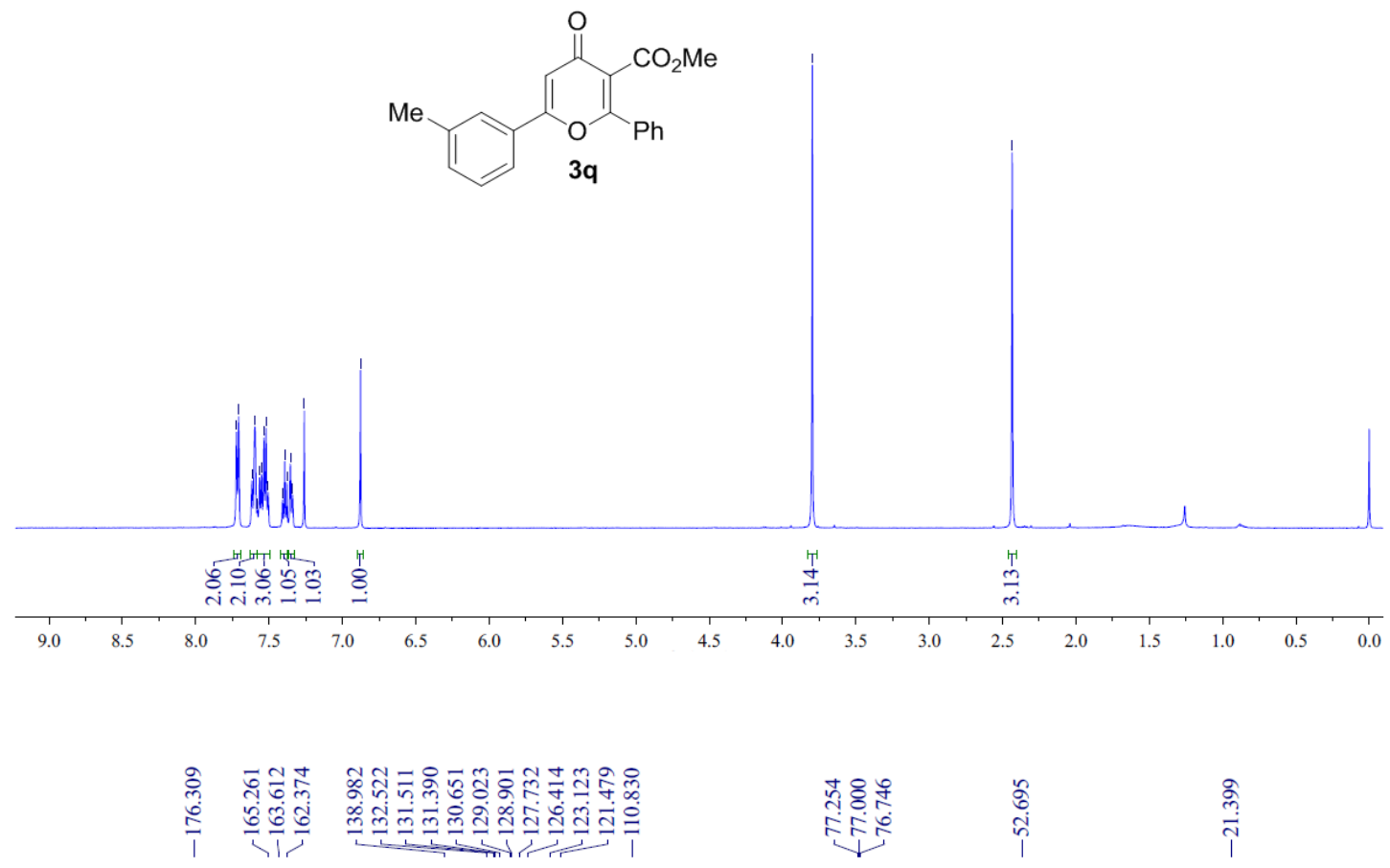<smiles>COC(=O)c1c(-c2ccccc2)oc(-c2cccc(C)c2)cc1=O</smiles>

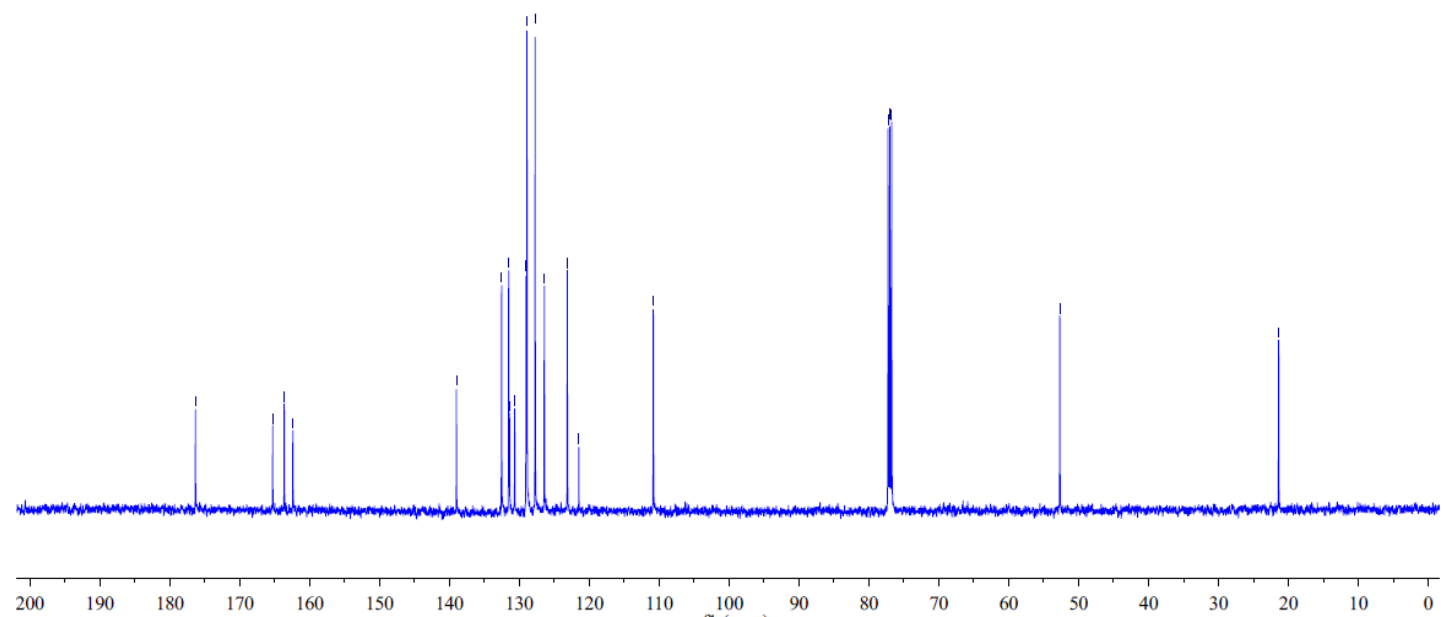



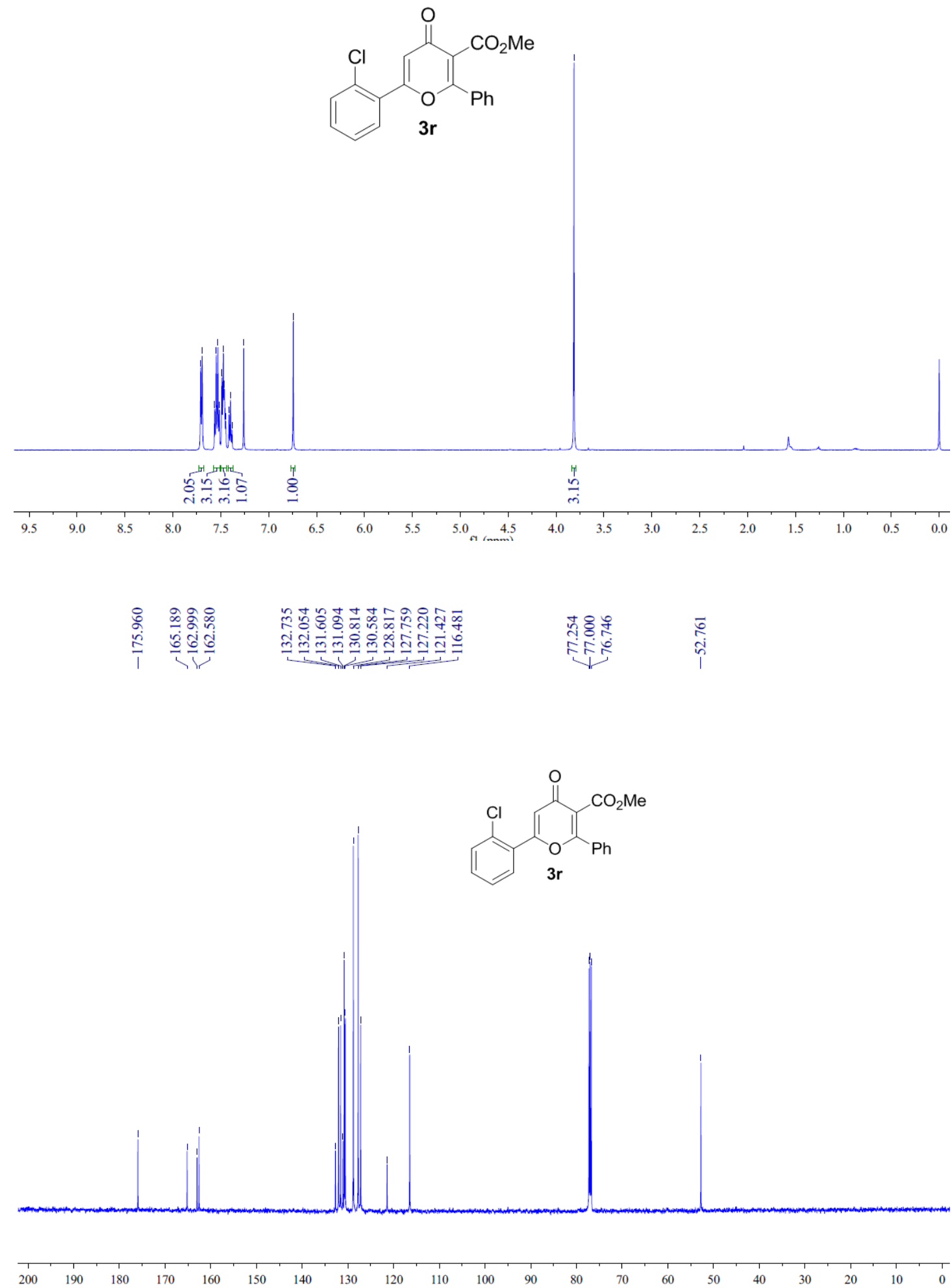

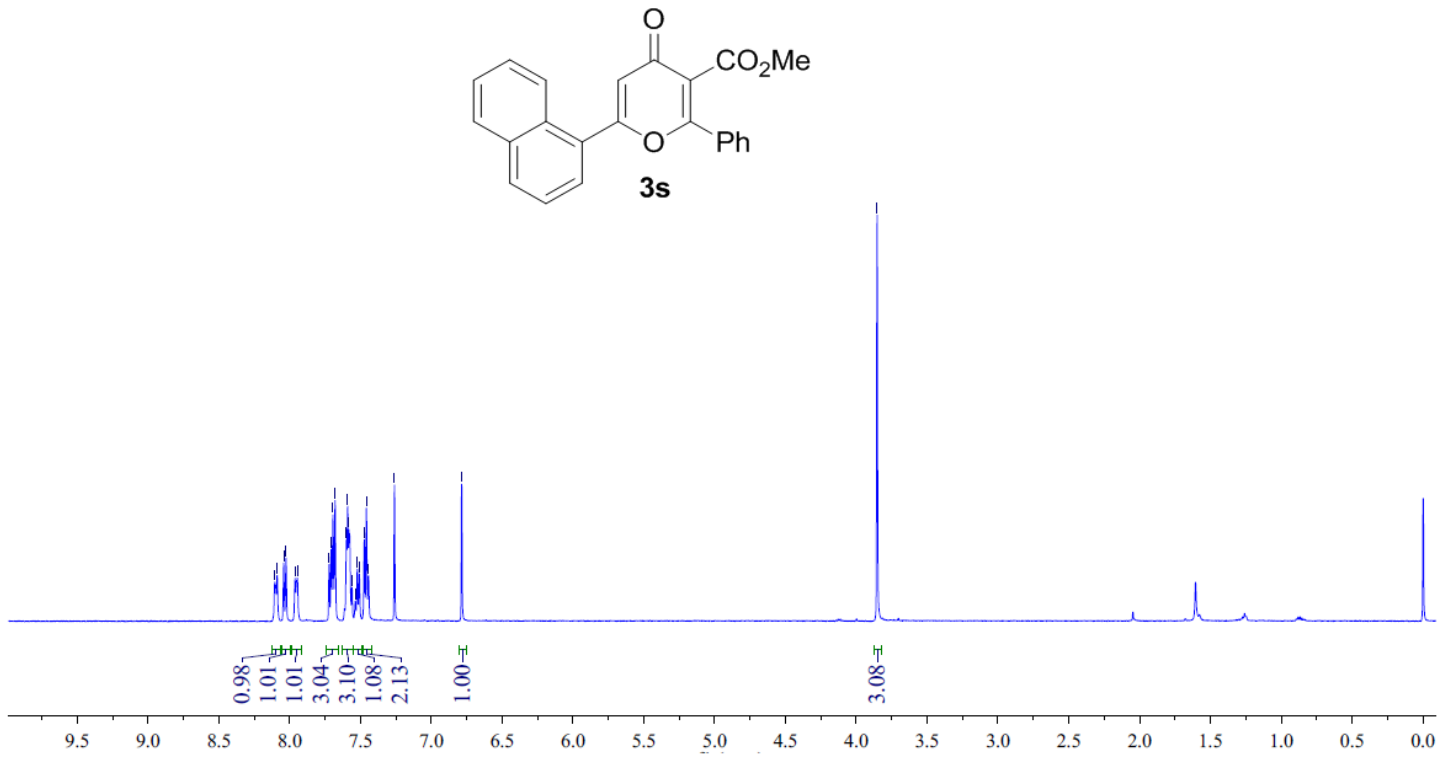

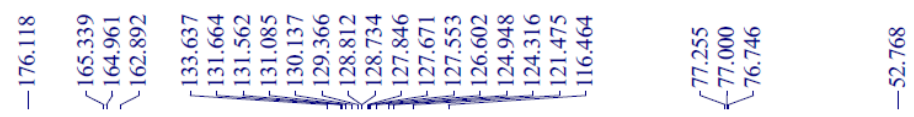

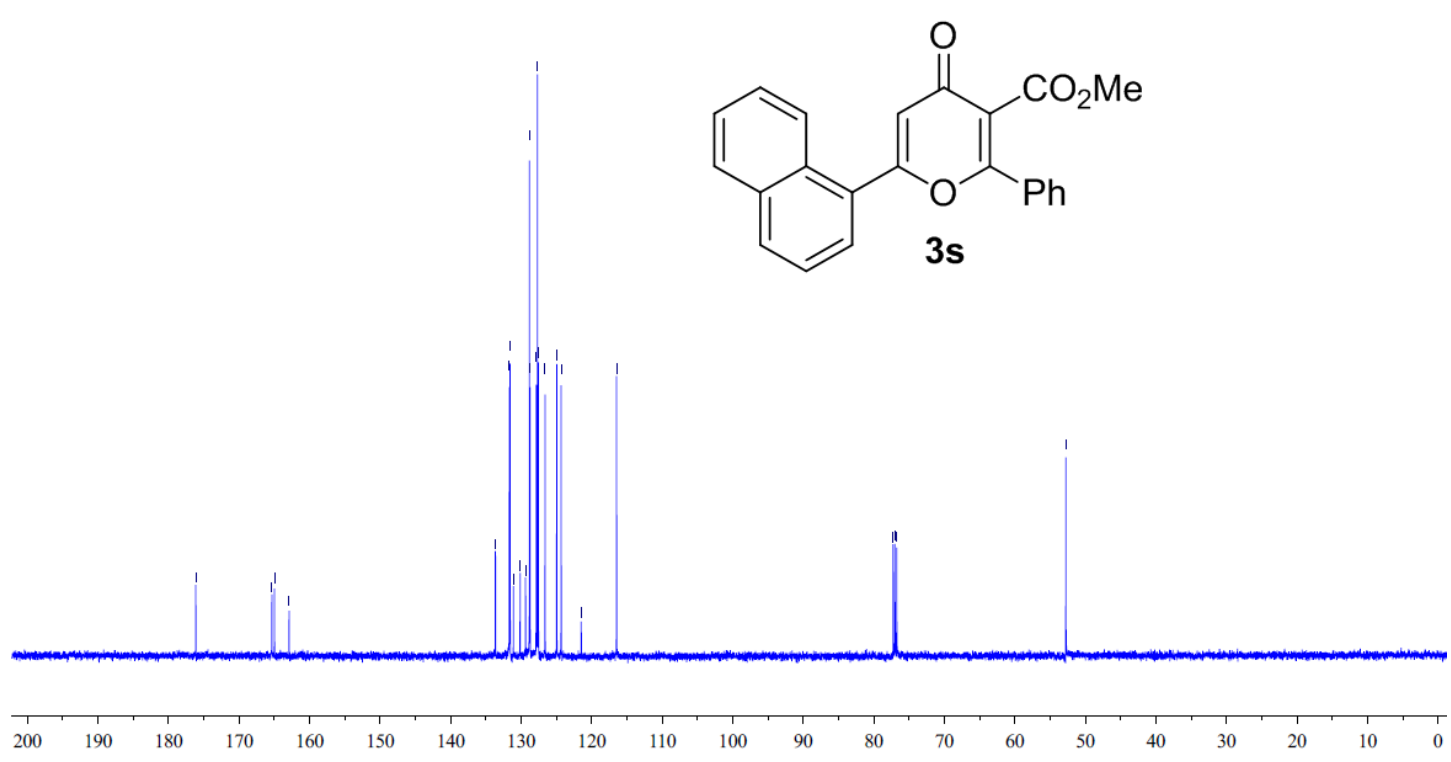



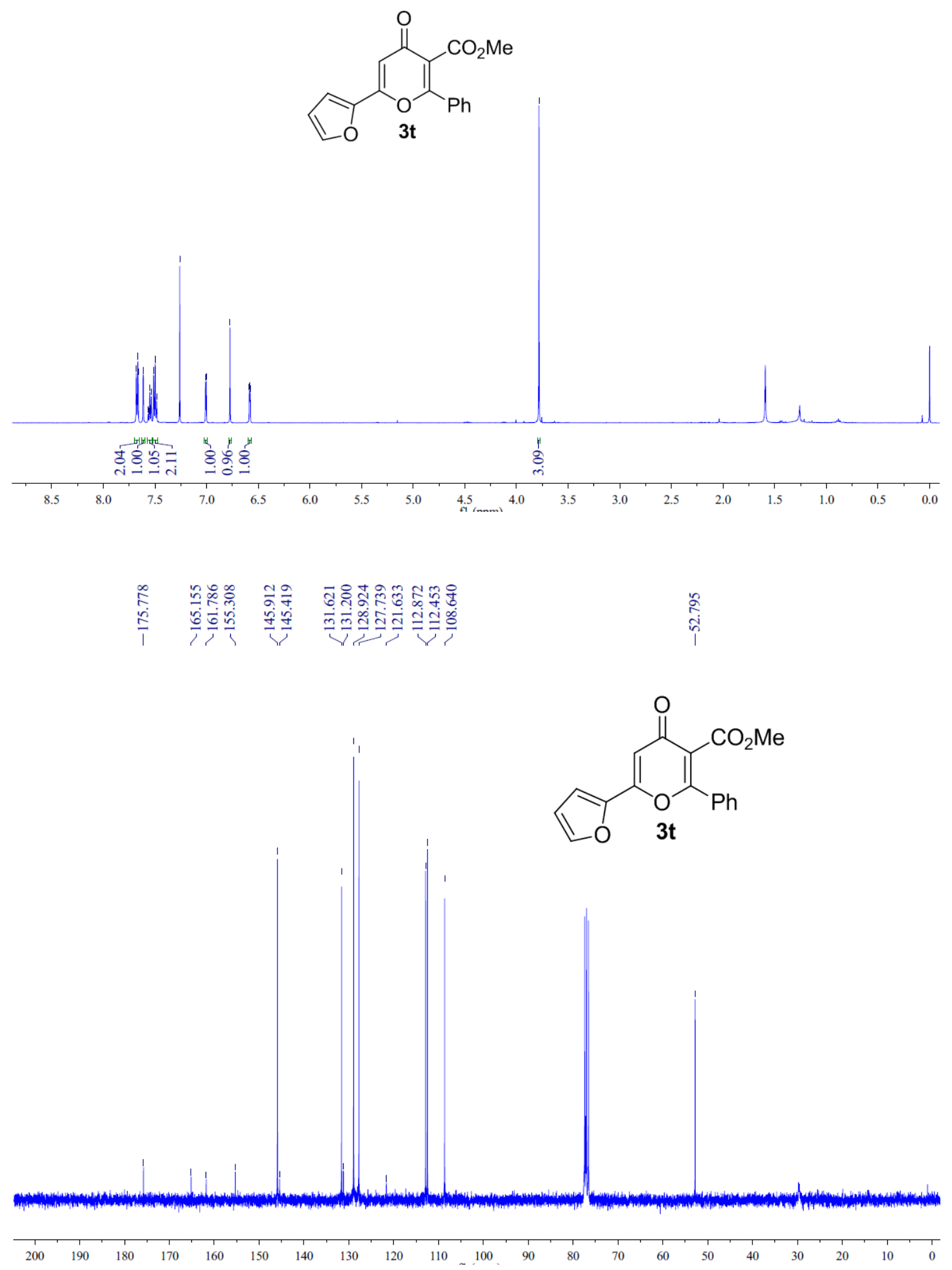

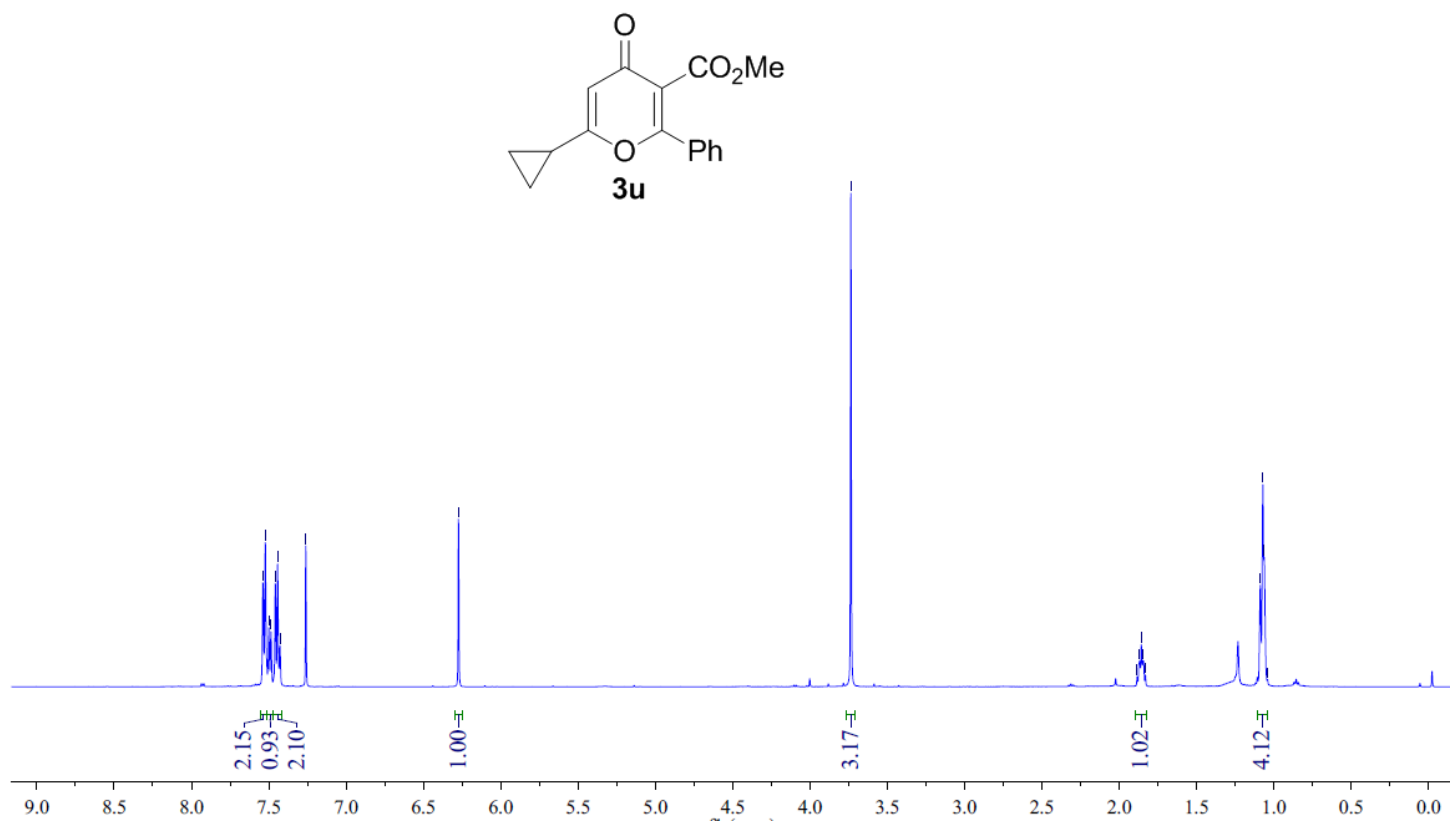

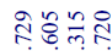

응

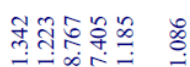

> \द



के

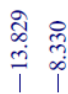

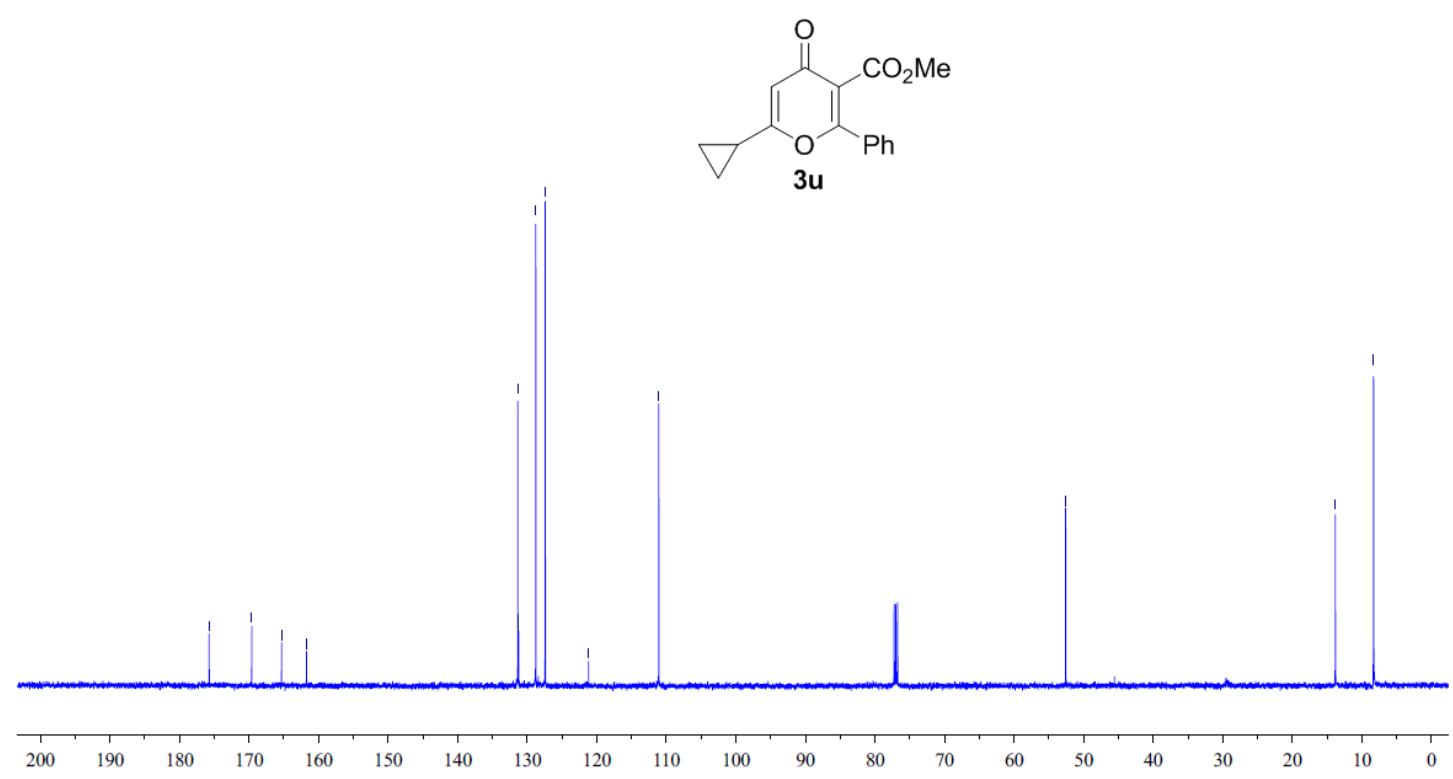



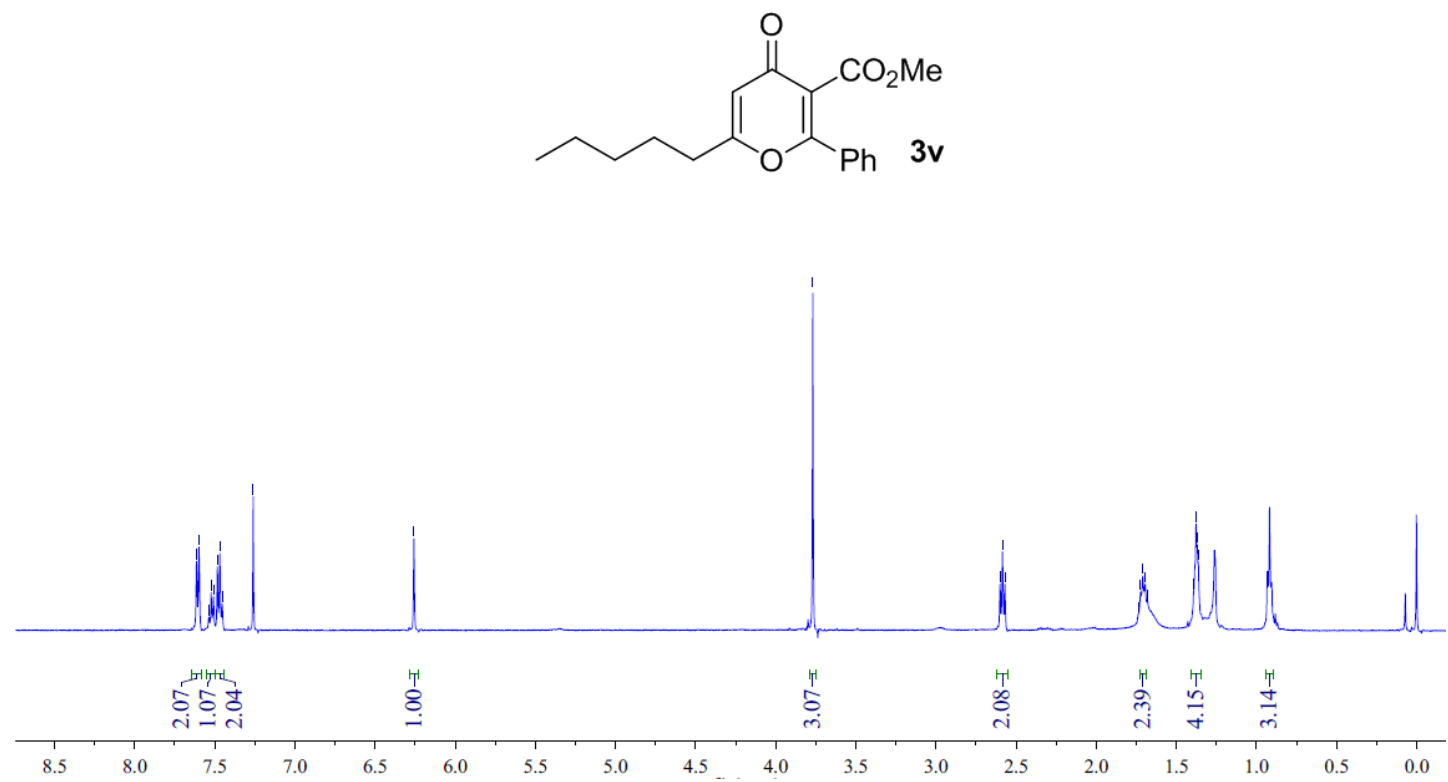

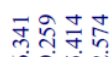

은

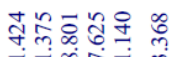

뷔ㅇㅝㅝ

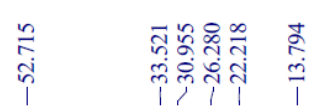<smiles>CCCCCc1cc(=O)c(C(C)=O)c(-c2ccccc2)o1</smiles>

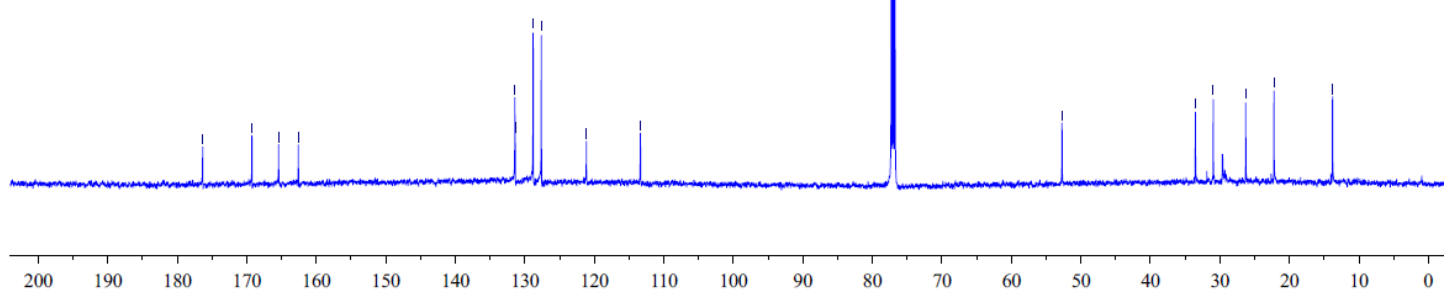



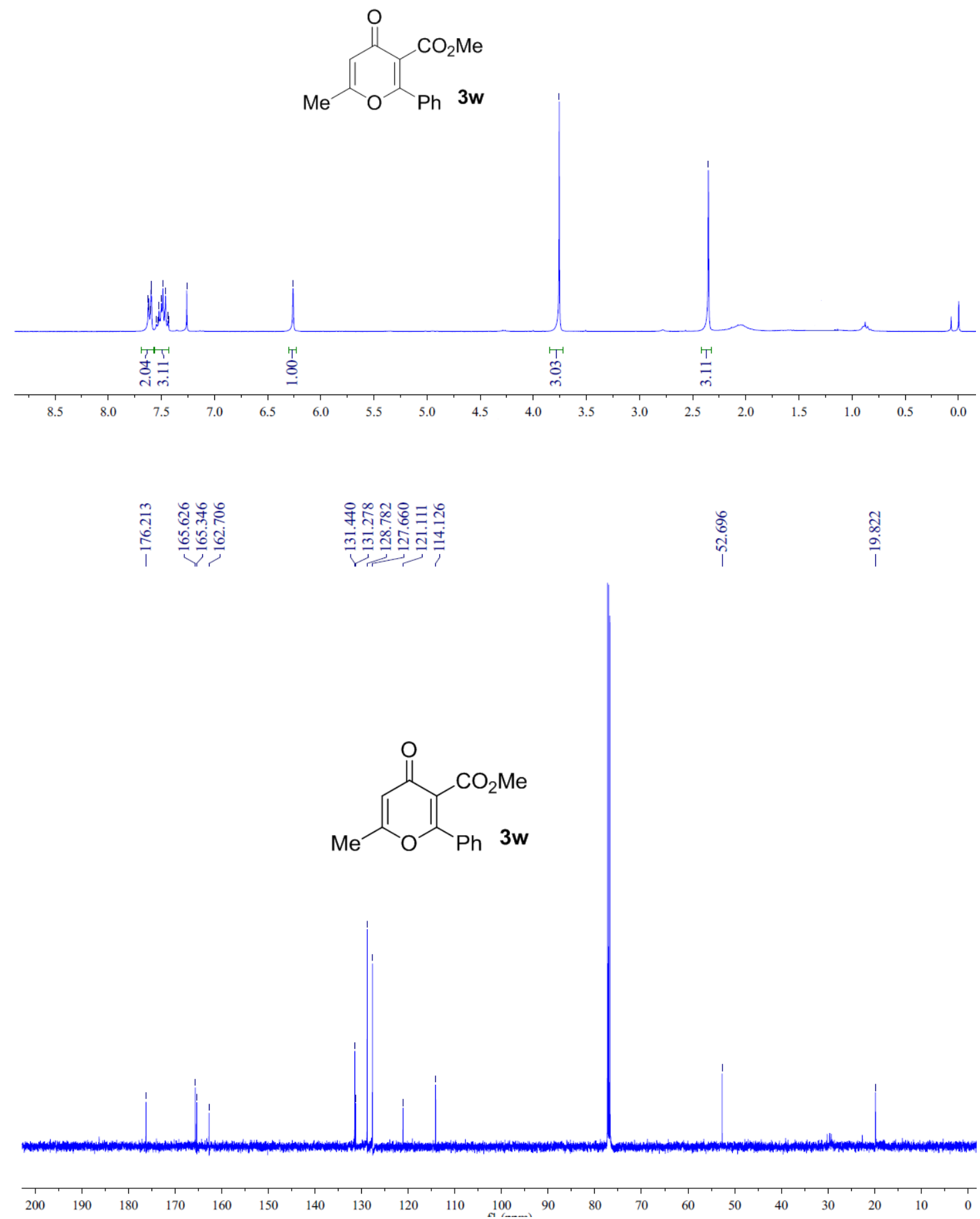

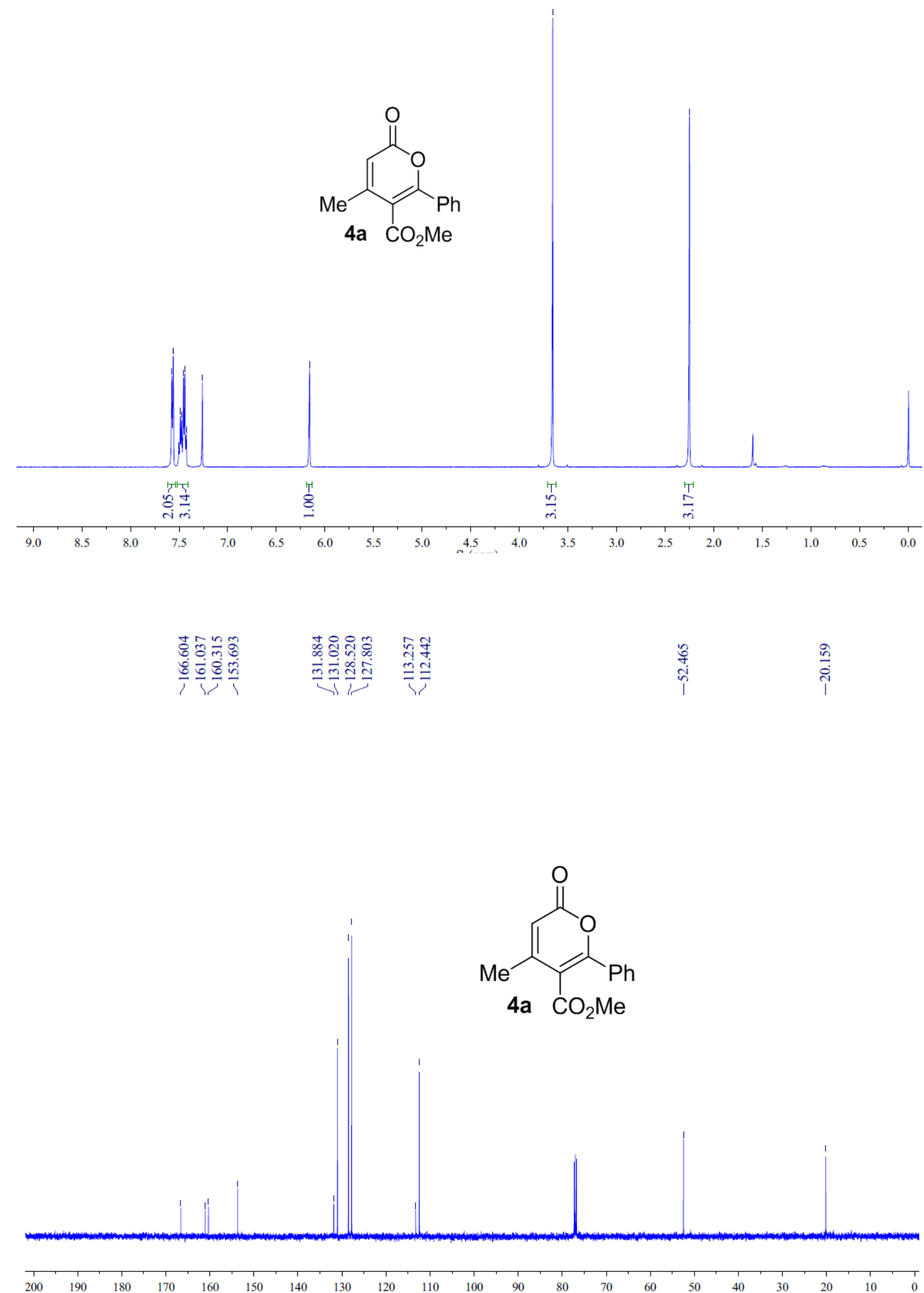

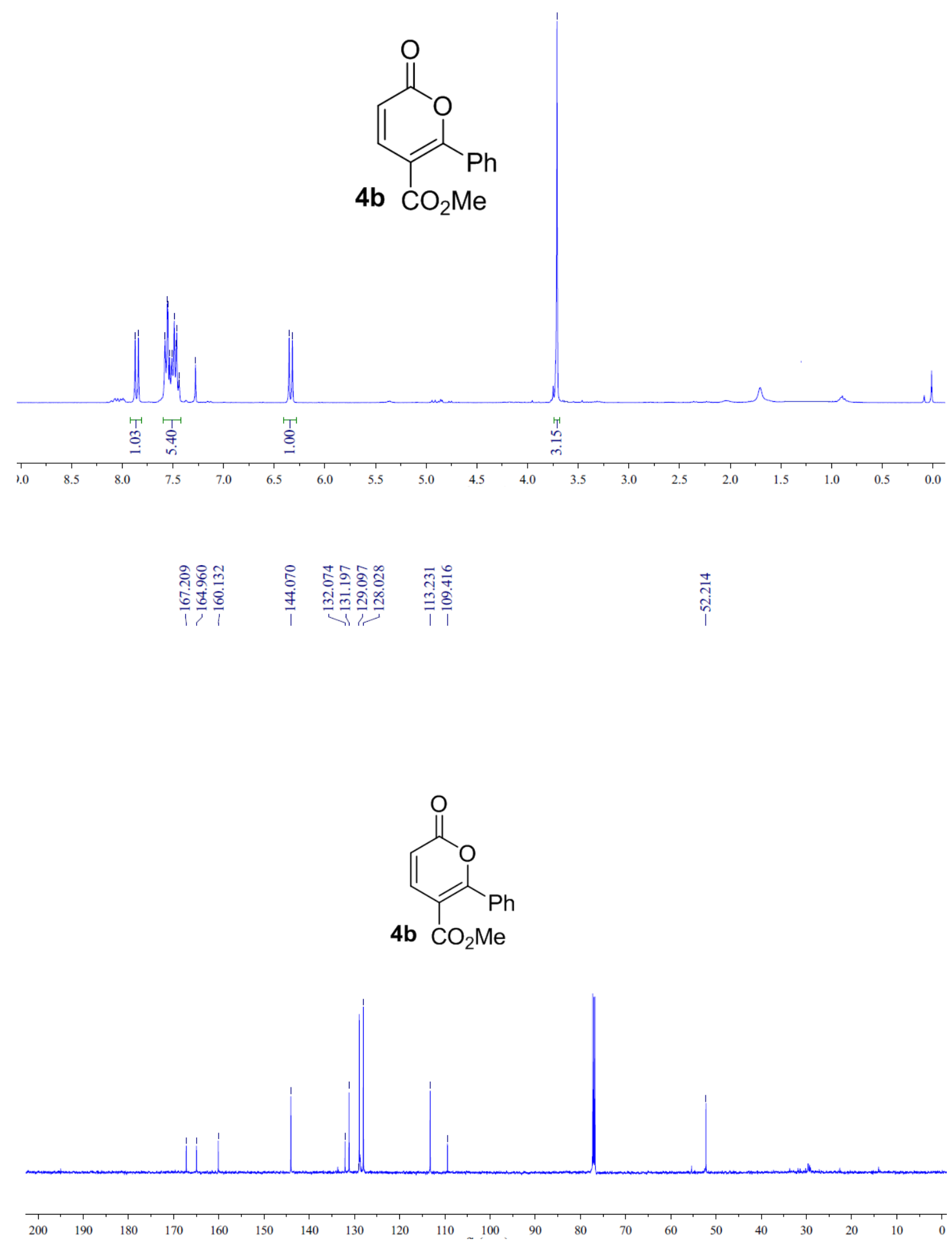


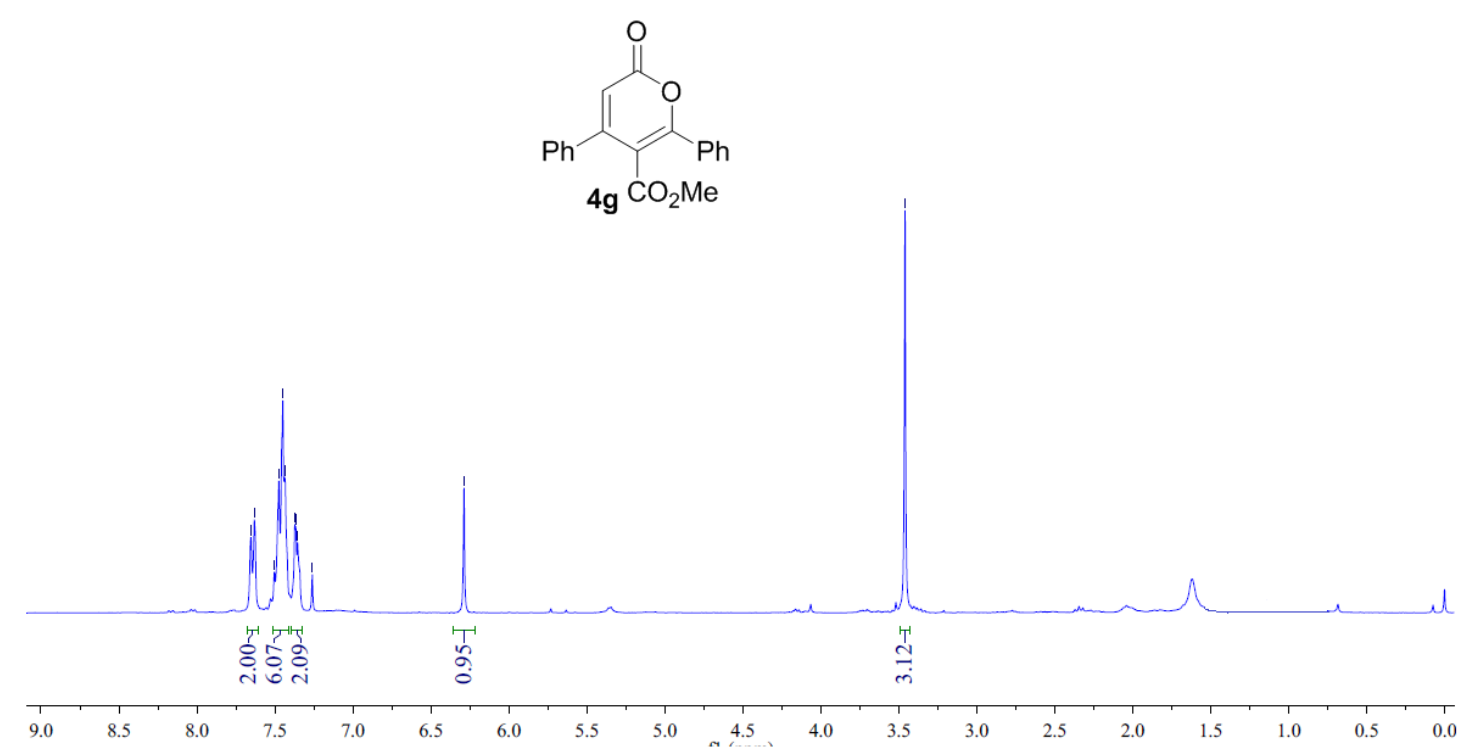



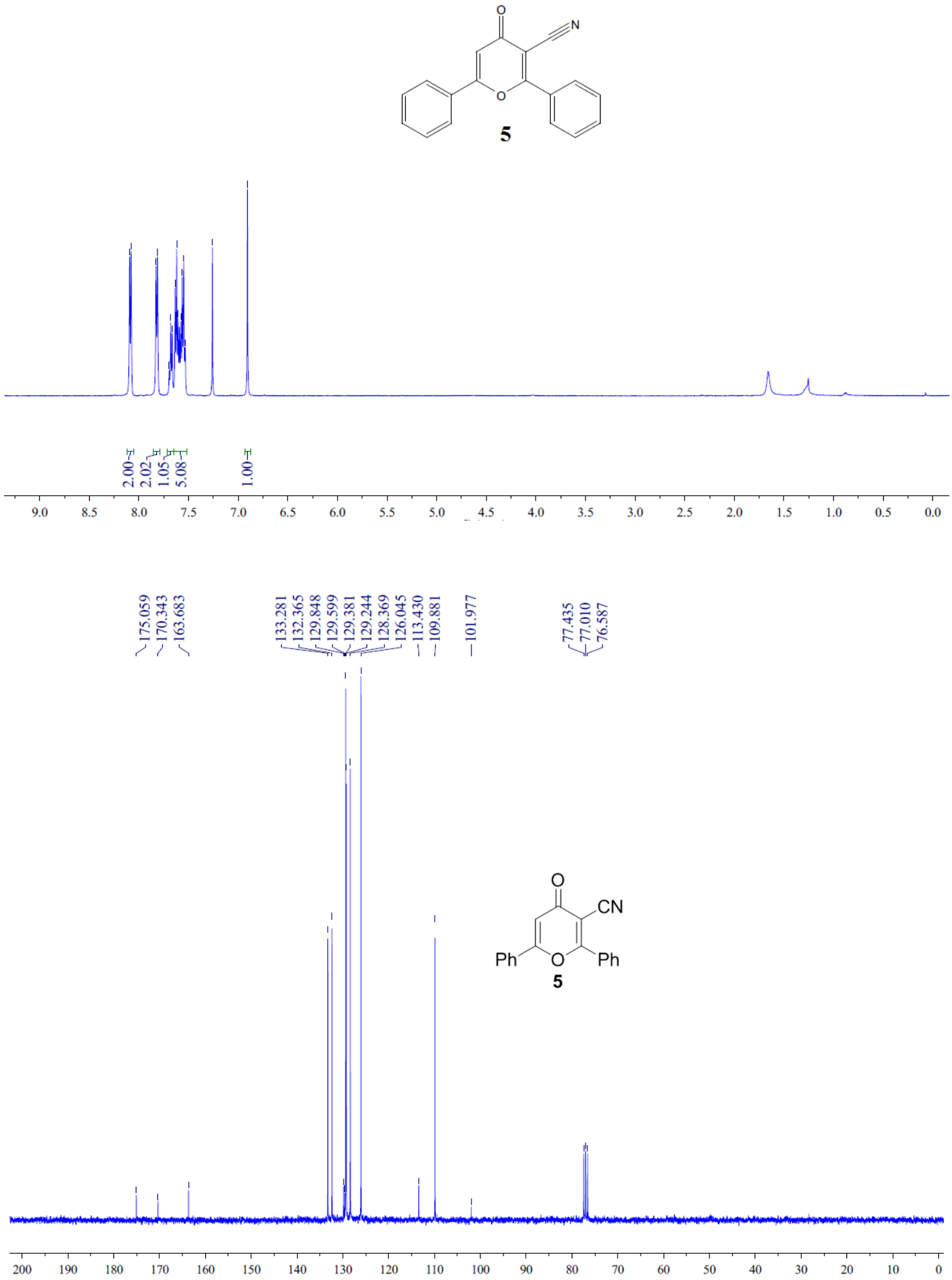

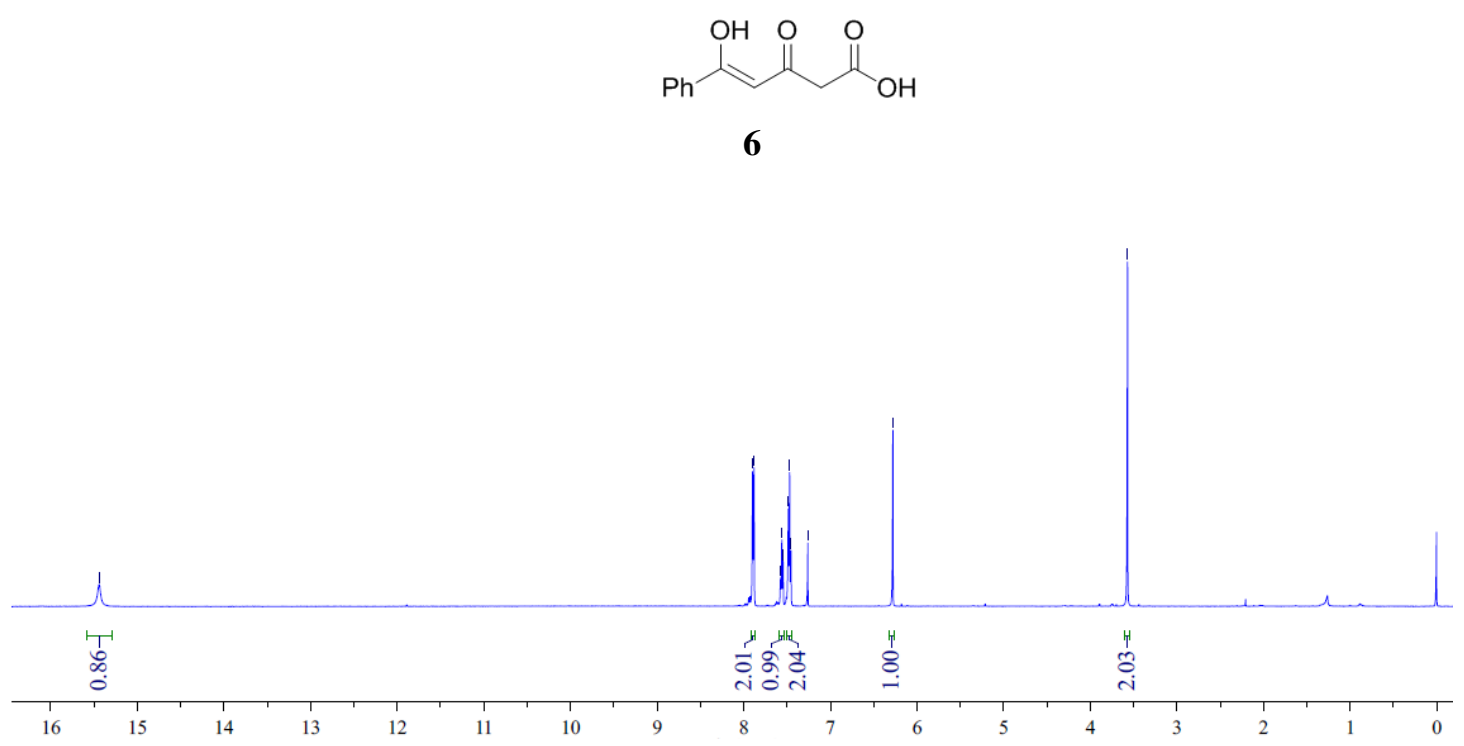


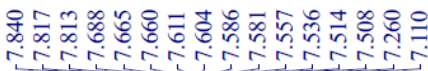


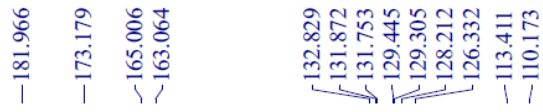

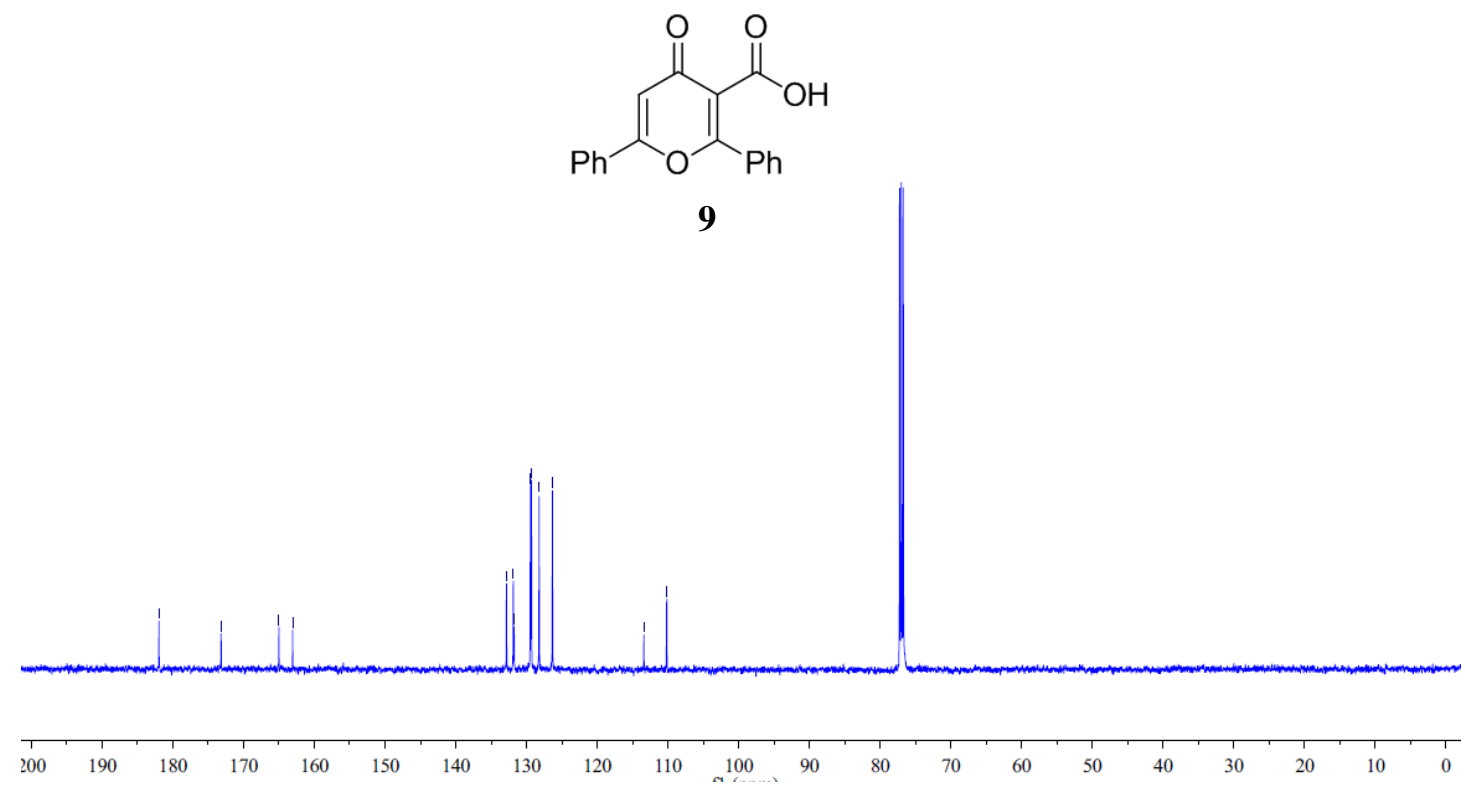



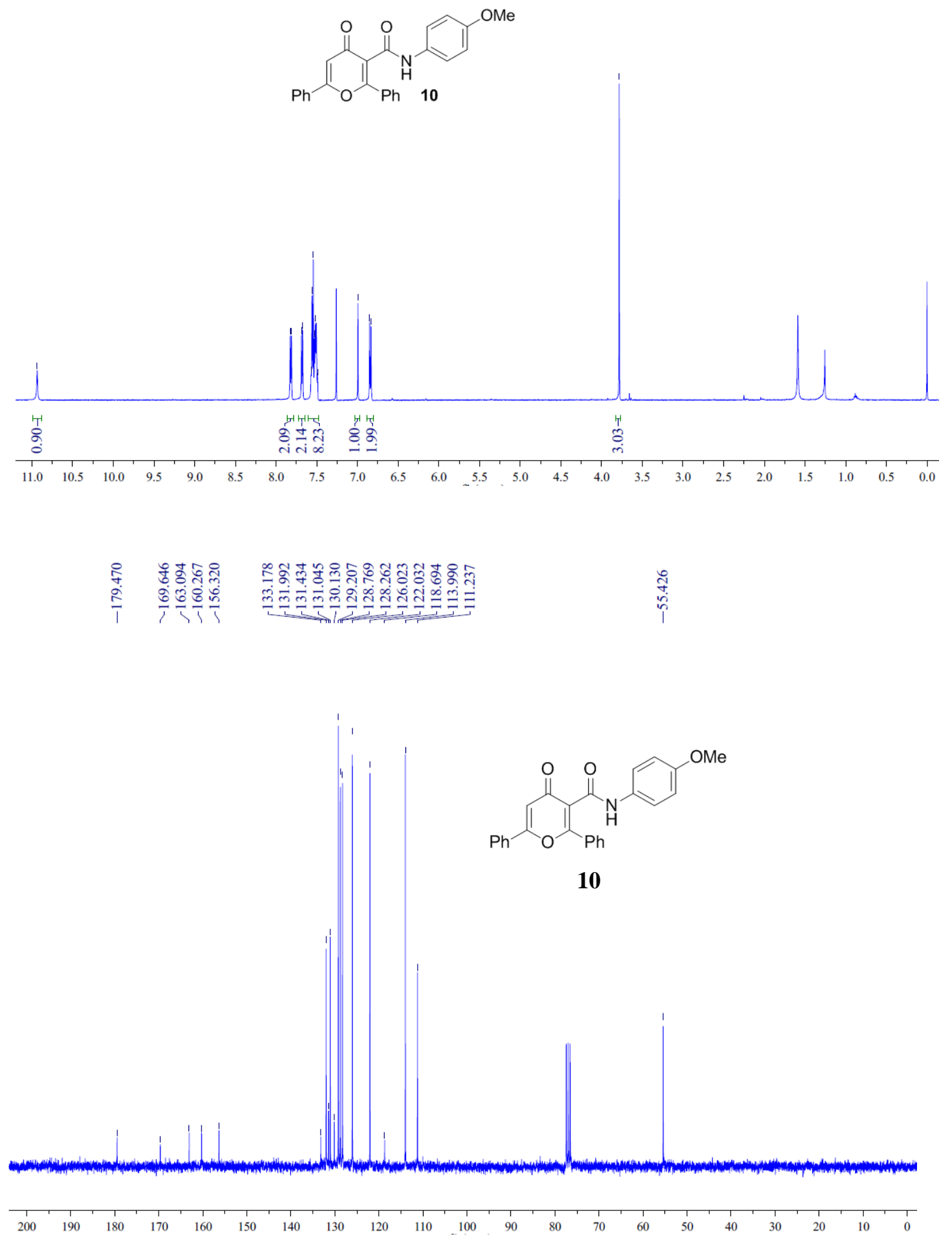

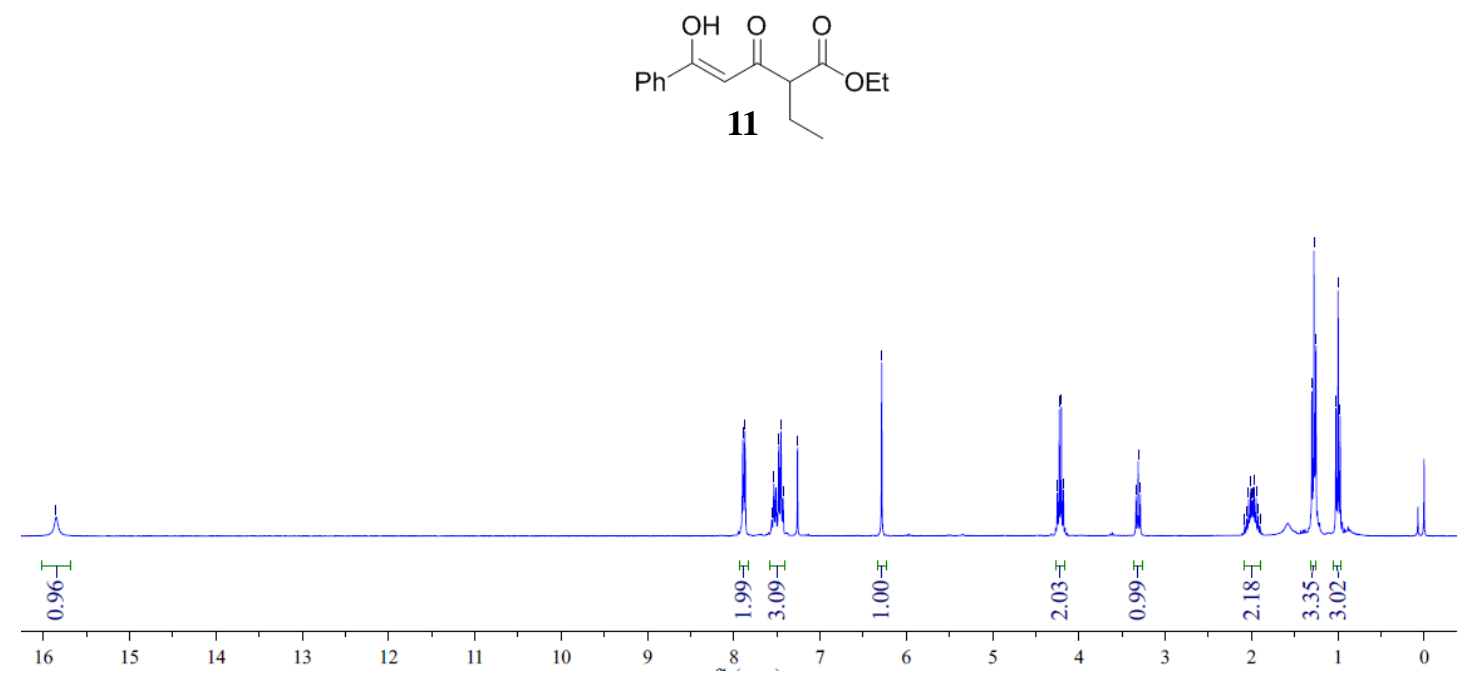

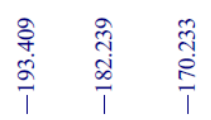

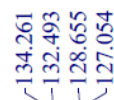

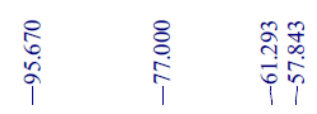

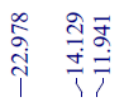

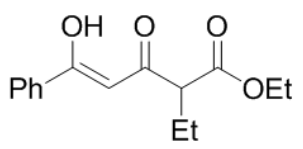

11

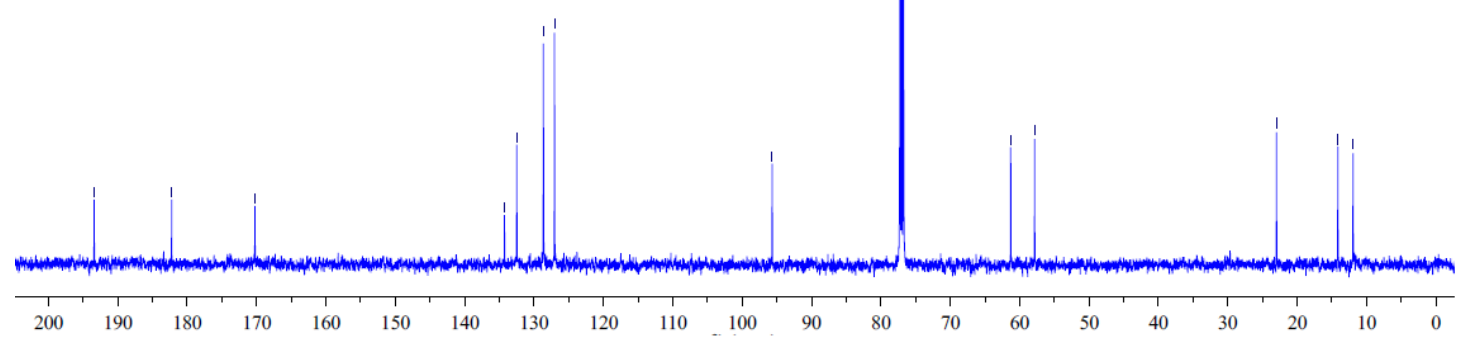

